\title{
Oxidative stress in vagal neurons promotes parkinsonian pathology and intercellular $\alpha$-synuclein transfer
}

\author{
Ruth E. Musgrove, ${ }^{1}$ Michael Helwig, ${ }^{1}$ Eun-Jin Bae, ${ }^{1,2}$ Helia Aboutalebi, ${ }^{1}$ Seung-Jae Lee, ${ }^{2}$ Ayse Ulusoy, ${ }^{1}$ and Donato A. Di Monte ${ }^{1}$ \\ ${ }^{1}$ Cerman Center for Neurodegenerative Diseases (DZNE), Bonn, Germany. ${ }^{2}$ Departments of Biomedical Sciences and Medicine, Neuroscience Research Institute, Seoul National University College of Medicine,
} Seoul, South Korea.

\begin{abstract}
Specific neuronal populations display high vulnerability to pathological processes in Parkinson's disease (PD). The dorsal motor nucleus of the vagus nerve $(\mathrm{DMnX})$ is a primary site of pathological $\alpha$-synuclein deposition and may play a key role in the spreading of $\alpha$-synuclein lesions within and outside the CNS. Using in vivo models, we show that cholinergic neurons forming this nucleus are particularly susceptible to oxidative challenges and accumulation of ROS. Targeted $\alpha$-synuclein overexpression within these neurons triggered an oxidative stress that became more pronounced after exposure to the ROSgenerating agent paraquat. A more severe oxidative stress resulted in enhanced production of oxidatively modified forms of $\alpha$-synuclein, increased $\alpha$-synuclein aggregation into oligomeric species, and marked degeneration of $\mathrm{DMnX}$ neurons. Enhanced oxidative stress also affected neuron-to-neuron protein transfer, causing an increased spreading of $\alpha$-synuclein from the $\mathrm{DMnX}$ toward more rostral brain regions. In vitro experiments confirmed a greater propensity of $\alpha$-synuclein to pass from cell to cell under prooxidant conditions and identified nitrated $\alpha$-synuclein forms as highly transferable protein species. These findings substantiate the relevance of oxidative injury in PD pathogenetic processes, establish a relationship between oxidative stress and vulnerability to $\alpha$-synuclein pathology, and define a mechanism, enhanced cell-to-cell $\alpha$-synuclein transmission, by which oxidative stress could promote PD development and progression.
\end{abstract}

\section{Introduction}

Oxidative stress has long been implicated in the pathogenesis of Parkinson's disease (PD), the second most common human neurodegenerative disorder. Pathological hallmarks of PD are the degeneration of discrete neuronal populations and progressive accumulation of $\alpha$-synuclein-containing intraneuronal inclusions called Lewy bodies and Lewy neurites. Initial evidence linking oxidative stress to selective neurodegeneration focused on dopaminergic neurons in the substantia nigra pars compacta and led to the notion that their high vulnerability to PD pathology was due, at least in part, to a prooxidant environment generated by reactions involving dopamine itself $(1,2)$. More recent experimental work has elucidated a cascade of toxic events that could further explain the severe loss of dopaminergic cells and pronounced buildup of nigral Lewy inclusions seen in PD. A cell-autonomous pacemaking activity was found to cause large oscillations in intracellular calcium concentration within vulnerable nigral neurons, leading to mitochondrial oxidant stress, dopamine oxidation, lysosomal dysfunction, and $\alpha$-synuclein accumulation (3-5). Once $\alpha$-synuclein burden is enhanced within dopaminergic cells, a vicious cycle may be set in motion, since increased $\alpha$-synuclein could promote the

Related Commentary: p. 3530

Conflict of interest: SJL is a founder and CEO of Neuramedy Ltd. Copyright: (5) 2019, American Society for Clinical Investigation. Submitted: January 16, 2019; Accepted: June 6, 2019; Published: August 5, 2019 Reference information: / Clin Invest. 2019;129(9):3738-3753. https://doi.org/10.1172/JCl127330. formation of protein aggregates and these aggregates may in turn exacerbate oxidative stress $(6,7)$.

Besides nigral dopaminergic cells, other neuronal populations are targeted by PD pathology. However, the role of oxidative stress as a mechanism contributing to this extranigral susceptibility and its involvement in pathogenetic processes that affect neurons devoid of dopamine content remain relatively unclear. In this study, the relationship among oxidative stress, neurodegeneration, and $\alpha$-synuclein pathology was investigated in vivo in the mouse dorsal motor nucleus of the vagus (Xth) nerve (DMnX). Several lines of consideration underscore the rationale for choosing this specific brain region. The DMnX contains cholinergic neurons that are vulnerable to degeneration in PD (8-10). Similarly to nigral dopaminergic cells, DMnX neurons are spontaneously active; they exhibit autonomous pacemaking activity that is associated with a sustained calcium entry and high metabolic demands (11). The relevance of investigations focusing on $\mathrm{DMnX}$ neurons is further indicated by our current knowledge of $\alpha$-synuclein pathophysiology. In postmortem PD brain, DMnX neurons are primary sites of accumulation of Lewy inclusions and are affected by $\alpha$-synuclein aggregate pathology at the earliest stages of disease development (12). Pathological $\alpha$-synuclein can be transferred from neuron to neuron, and through this mechanism, $\alpha$-synuclein lesions may spread throughout the brain and reach vulnerable brain regions (13-16). As one of the initial sites of $\alpha$-synuclein pathology, the DMnX could therefore play a critical role in the early process of protein accumulation and interneuronal protein transmission. Finally, evidence of $\alpha$-synuclein lesions in the peripheral nervous system has prompted the suggestion that 
A

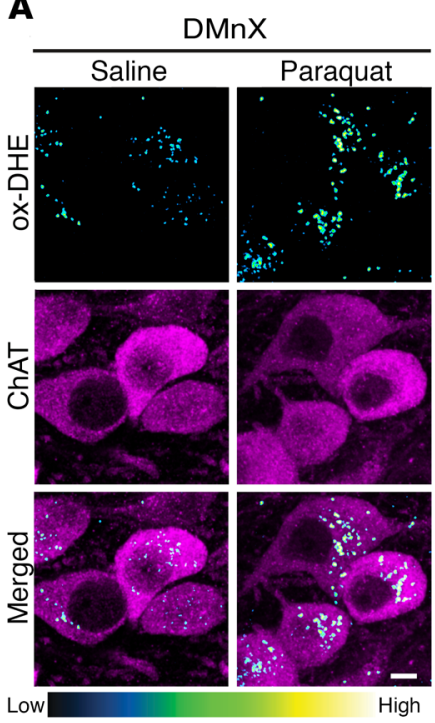

D

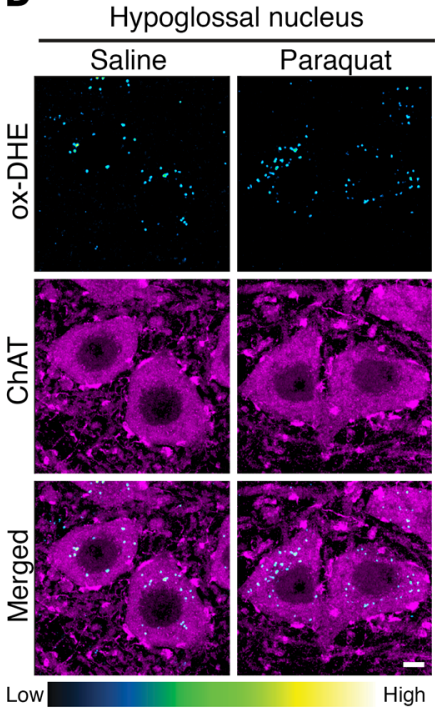

B

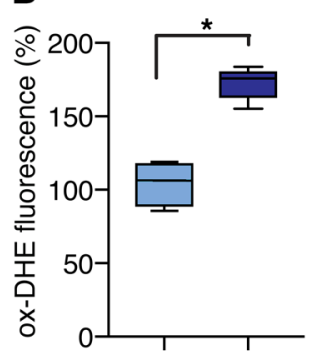

C

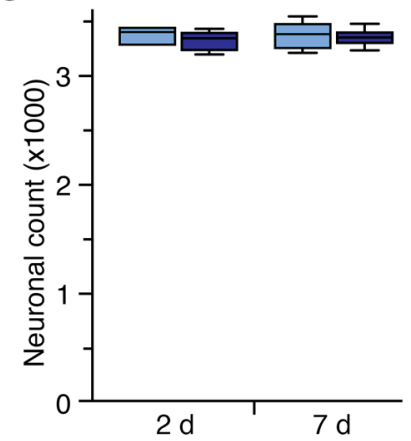

E

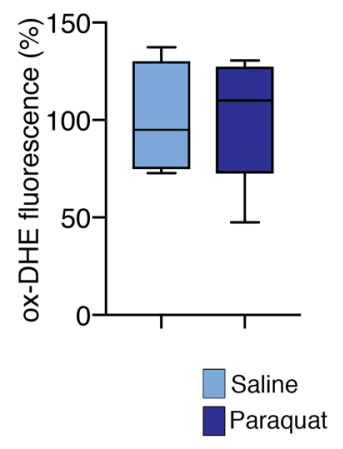

pathological forms of the protein may travel long distance from peripheral tissues to the brain and vice versa, from the brain to peripheral tissues (16). Both clinical and experimental observations support the likelihood that DMnX neurons, with their long visceromotor projections, represent key carriers of $\alpha$-synuclein pathology on this central-to-peripheral route (17-19).

In the present study, targeted accumulation of $\alpha$-synuclein within cholinergic DMnX neurons was achieved by a single injection of adeno-associated viral vectors (AAVs) carrying human $\alpha$-synuclein (ho-synuclein) DNA into the mouse vagus nerve. Features of this model include aggregation and neuron-to-neuron transmission of h $\alpha$-synuclein as well as selective degeneration of the overexpressing DMnX cells $(20,21)$. To induce oxidative stress both in vivo and in vitro, mice and cells in culture were treated with paraquat, a bipirydyl agent capable of generating substantial amounts of ROS via redox cycling with molecular oxygen (22). In vivo results revealed pronounced vulnerability of DMnX cholinergic neurons to oxidative challenges. Severe oxidative stress, as induced by paraquat exposure together with $\alpha$-synuclein overex-

Figure 1. Paraquat-induced oxidative stress causes ROS accumulation in the DMnX, but not the hypoglossal nucleus. (A) Mice received 2 i.p. injections of either saline or paraquat separated by a 1-week interval and were sacrificed at 2 days after treatment. They were also injected with DHE 1 hour before the time of sacrifice. Representative confocal images show fluorescent puncta of ox-DHE (a marker of ROS formation, blue-greenyellow color graded) within ChAT-immunoreactive neurons (magenta) in the DMnX. Scale bar: $5 \mu \mathrm{m}$. (B) Comparison of the integrated density of fluorescent ox-DHE puncta within ChAT-positive $\mathrm{DMnX}$ neurons from mice treated with saline ( $n=4$, light blue bar) or paraquat ( $n=5$, dark blue bar). Approximately 100 neurons/animal were analyzed and averaged. Values were calculated as percentage of the mean value in saline-injected animals. (c) Mice injected with saline ( $n=4 /$ time point) or paraquat ( $n=4$ /time point) were sacrificed at 2 and 7 days after treatment, and the number of Nissl-stained neurons was counted unilaterally in the $\mathrm{DMnX}$. (D) Representative confocal images show ChAT-positive hypoglossal neurons (magenta) containing fluorescent ox-DHE from mice injected with saline or paraquat and sacrificed 2 days after treatment. Scale bar: $5 \mu \mathrm{m}$. (E) Integrated density of ox-DHE puncta within ChAT-positive neurons in the hypoglossal nucleus. Analyses were carried out on tissue sections from mice treated with saline $(n=4)$ or paraquat $(n=5)$. Approximately 30 neurons/animal were analyzed and averaged. Values were calculated as percentage of the mean value in saline-injected animals. Box and whisker plots show median (middle line), upper and lower quartiles, and maximum and minimum as whiskers. ${ }^{*} P \leq 0.05$, Mann-Whitney $U$ test.

pression, caused an accumulation of oxidatively modified forms of $\alpha$-synuclein, enhanced protein aggregation, and marked neurodegeneration, indicating a contribution of oxidative stress to PD-relevant pathological processes in the DMnX. Quite remarkably, both in vivo and in vitro evidence also revealed the ability of oxidative stress to promote cell-to-cell $\alpha$-synuclein transfer and supported the conclusion that oxidized/nitrated forms of the protein are characterized by pronounced cell-to-cell mobility.

\section{Results}

Vulnerability of DMnX neurons to oxidative stress. Oxidative stress was compared in the DMnX of mice that received 2 i.p. injections (separated by a 1-week interval) of either vehicle (saline) or paraquat $(15 \mathrm{mg} / \mathrm{kg})$ and were sacrificed at 2 days after the second administration. To visualize and quantify ROS formation, the superoxide indicator dihydroethidium (DHE) was injected s.c. shortly before the time of sacrifice. Reaction of DHE with superoxide generates the fluorescent ethidium cation (ox-DHE); this cation can then be accumulated into mitochondria via the mitochondrial transmembrane potential, generating a punctate pattern of intracellular fluorescent signal $(23,24)$. Punctate fluorescence was observed within choline acetyltransferase-immunoreactive (ChAT-immunoreactive) DMnX neurons in sections of the mouse medulla oblongata from both control and paraquat-exposed mice; histology also revealed, however, that the number and intensity of labeled puncta were markedly augmented in specimens from the latter group of animals (Figure 1A). Intraneuronal quantification of the ethidium-generated fluorescent signal showed a significantly higher integrated density after paraquat administration, consistent with an increase in ROS formation (Figure 1B). Oxidative stress was further investigated by measurements of malondialdehyde, a marker of lipid peroxidation, in tissue specimens of the dorsal medulla oblongata. A prooxidant effect was indicated by a significant increase in malondialdehyde levels in samples 
A

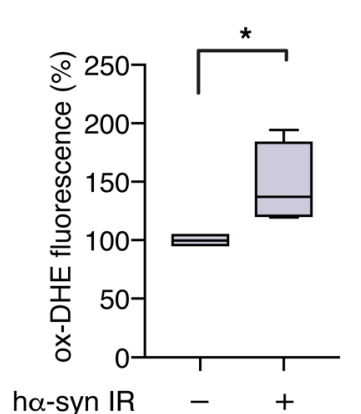

B

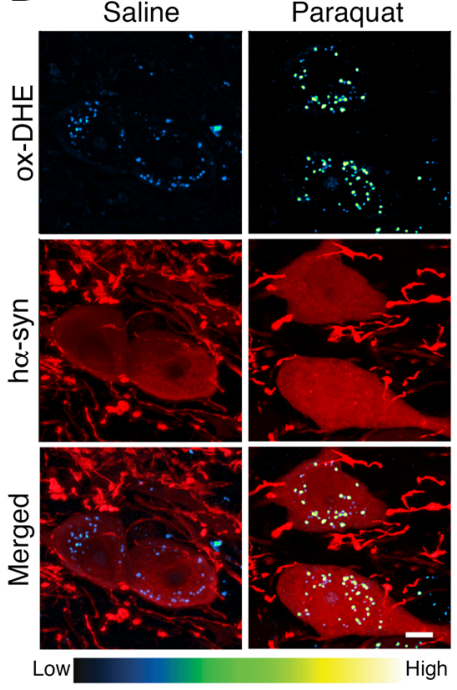

C

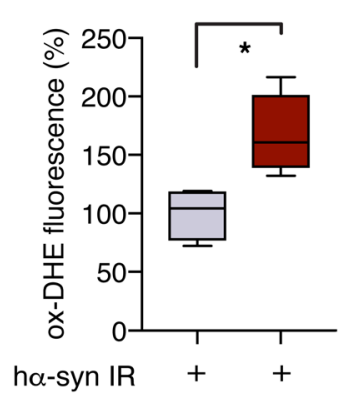

D

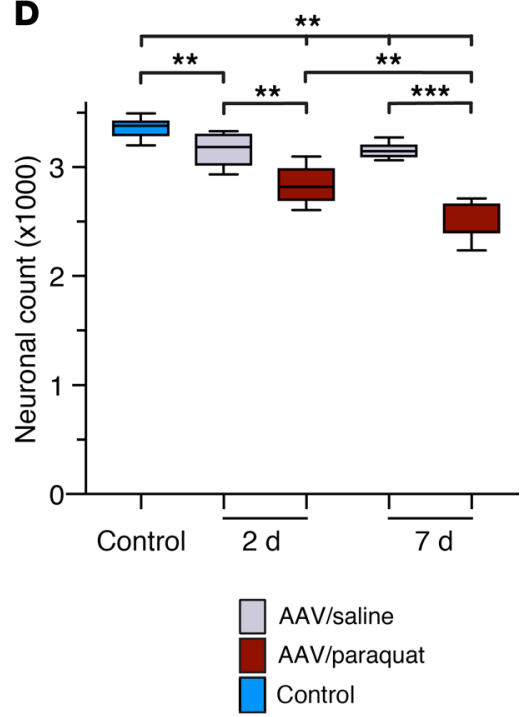

Figure 2. h $\alpha$-Synuclein overexpression causes an oxidative stress that is augmented by paraquat administration. $(\mathbf{A})$ Mice $(n=4)$ received a unilateral (left) intravagal injection of h $\alpha$-synuclein-carrying AAVs. They were then treated with $2 \mathrm{i}$.p. injections of saline and were sacrificed at 2 days after the second saline administration. They also received an injection of DHE 1 hour before the time of sacrifice. Ox-DHE fluorescent signal was compared in the left $\mathrm{DMnX}$ between transduced neurons immunoreactive for h $\alpha$-synuclein (h $\alpha$-syn IR) and neurons devoid of h $\alpha$-synuclein immunoreactivity. Approximately 50 neurons/animal were analyzed and averaged. Values were calculated as percentage of the mean value in h $\alpha$-synuclein-devoid cells. (B and C) h $\alpha$-Synuclein AAV-injected mice were treated with saline $(n=4)$ or paraquat $(n=5)$ and sacrificed at 2 days. They also received a DHE injection. (B) Representative confocal images show h $\alpha$-synuclein-positive neurons (red) containing fluorescent ox-DHE (blue-green-yellow) in the left DMnX. Scale bar: $5 \mu \mathrm{m}$. (C) Integrated density of ox-DHE fluorescence within h $\alpha$-synuclein-immunoreactive neurons in the left DMnX of mice treated with saline (gray bar) or paraquat (red bar). Approximately 50 neurons/animal were analyzed and averaged. Values were calculated as percentage of the mean value in h $\alpha$-synuclein AAV/saline-injected animals. (D) h $\alpha$-Synuclein AAV-injected mice were treated with either saline or paraquat and sacrificed at 2 ( $n \geq 5 /$ treatment) or 7 ( $n=7 /$ treatment) days after treatment. A group of control animals ( $n=8$, light blue bar) were only injected with saline. The number of Nissl-stained neurons was counted stereologically in the left $\mathrm{DMnX}$. Box and whisker plots show median, upper and lower quartiles, and maximum and minimum as whiskers. ${ }^{*} P \leq 0.05 ;{ }^{* *} P \leq 0.01$; ${ }^{* * *} P \leq 0.001$, Mann-Whitney $U$ test (A and $\mathbf{C}$ ) or Kruskal-Wallis followed by Conover-Iman post hoc test (D).

from paraquat-injected as compared with saline-injected mice (Supplemental Figure 1). To assess the neurotoxic consequences of paraquat-induced oxidative stress, the number of Nissl-stained neurons was counted stereologically in the DMnX. Results showed no difference in cell counts between saline- and paraquat-treated mice at 2 days after the second saline or paraquat injection. Also, no significant difference in neuronal number between the 2 groups of animals was seen at a later time point, namely 7 days after administration (Figure 1C).

Susceptibility to oxidative stress was then evaluated within other populations of cholinergic cells. First, analyses focused on ChAT-positive neurons that lie immediately below the DMnX and constitute the hypoglossal nucleus. In findings similar to those in the DMnX, oxidation-induced DHE fluorescence was detected within hypoglossal neurons in saline- and paraquat-injected mice (Figure 1D). In contrast with data in the DMnX, however, histological observations and measurements of integrated fluorescence density showed no significant changes as a result of paraquat exposure (Figure 1, D and E). Next, analyses were carried out to assess ox-DHE fluorescence within striatal cholinergic interneurons and ChAT-positive cells in the medial septal nucleus. In both regions, data showed ROS levels that were similar in saline- and paraquat-treated animals (Supplemental Figure 2).

Oxidative stress and $\alpha$-synuclein burden in the DMnX. To investigate the relationship between $\alpha$-synuclein accumulation and oxidative stress, mice were first injected with ho-synuclein AAVs into the left vagus nerve. Then, 2 weeks later, at a time when transduction and overexpression of the exogenous protein are fully attained in the $\operatorname{DMnX}(20,25)$, they received 2 injections (at a 1-week interval) of either saline or paraquat. Sections of the medulla oblongata were immunostained with a specific antibody recognizing human but not rodent $\alpha$-synuclein (MJFR1). Counts of neurons positive and negative for h $\alpha$-synuclein showed that AAV-induced transduction caused overexpression in approximately $40 \%$ of neurons in the left DMnX (ipsilateral to the AAV injection); no ho-synucleincontaining cells were detected in the contralateral DMnX. Transduction efficiency was similar in control and paraquat-treated mice (data not shown). Oxidative stress was evaluated in animals sacrificed at 2 days after the second saline or paraquat administration; these mice also received a DHE injection prior to the time of sacrifice. To determine whether h $\alpha$-synuclein overexpression was itself capable of inducing oxidative stress, ethidium-generated fluorescence was quantified in AAV-injected animals treated with saline and compared between neurons devoid of detectable h $\alpha$ synuclein versus $h \alpha$-synuclein-containing neurons in the left DMnX. Results revealed that a higher fluorescent signal characterized the transduced cells (Figure 2A). To test the possibility that paraquat administration may induce a further increase in ROS production over the one caused by h $\alpha$-synuclein, fluorescence was compared between $\mathrm{h} \alpha$-synuclein-immunoreactive neurons in saline-treated mice and ho-synuclein-positive neurons in paraquat-injected animals. Paraquat exposure indeed triggered a 
A

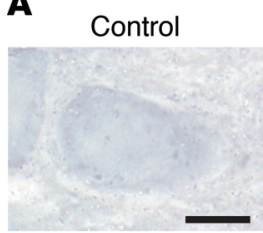

B

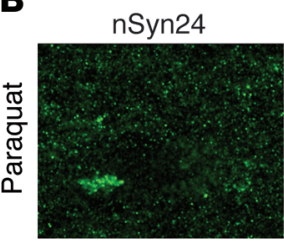

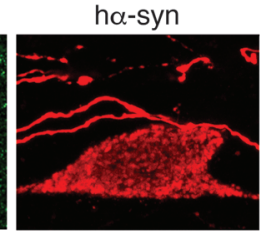

Saline
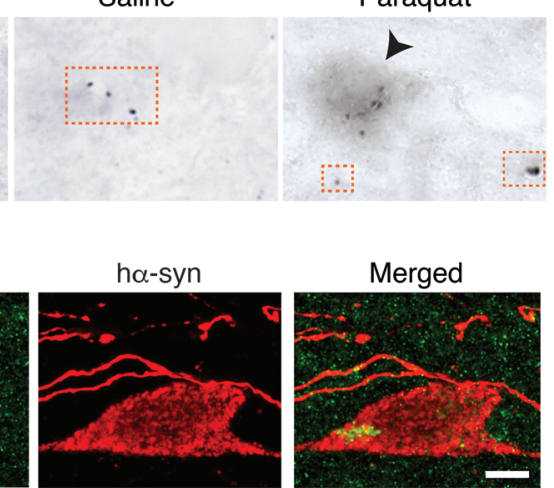

C

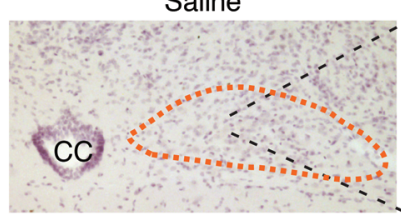

Paraquat

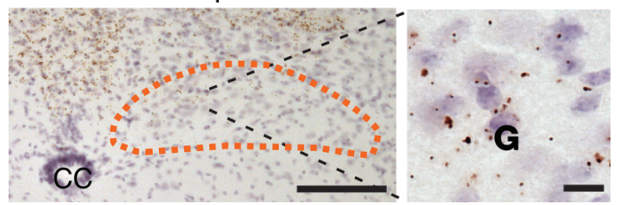

D

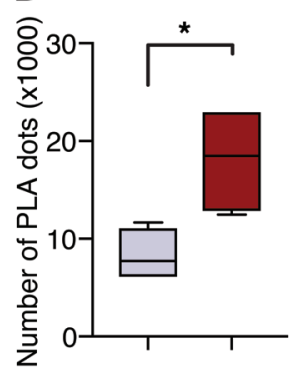

E
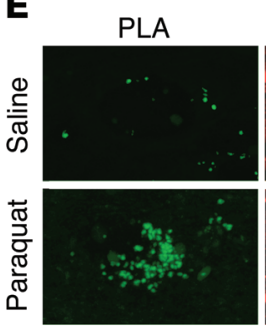
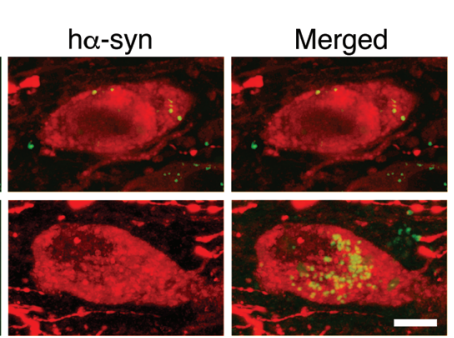

$\mathbf{F}$
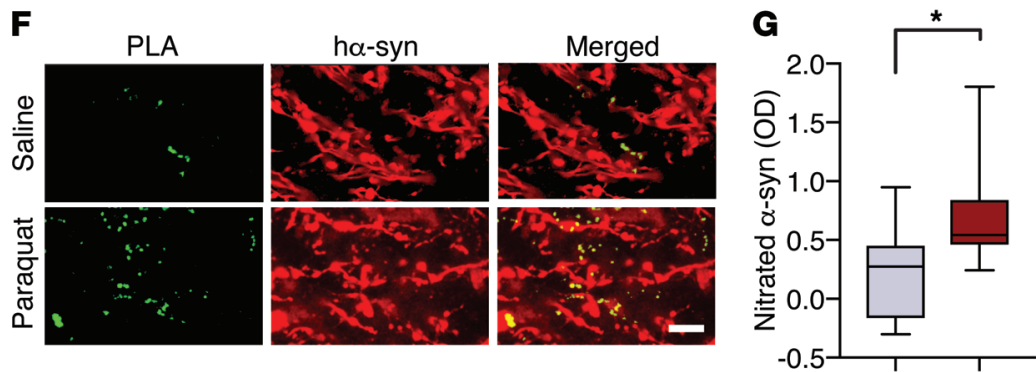

H
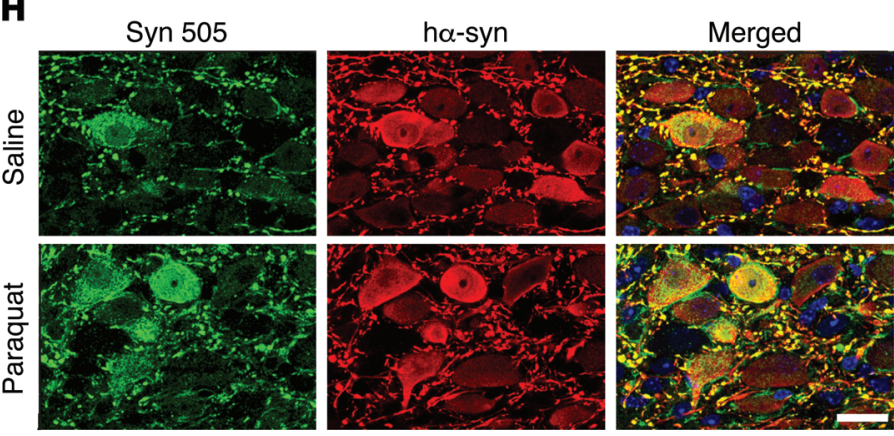

I

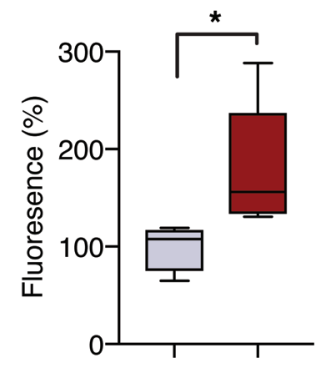

AAV/saline

AAV/paraquat

Figure 3. Severity of oxidative stress affects the degree of ho-synuclein oxidative modifications. Mice received an infusion of h $\alpha$-synuclein-carrying AAVs into the left vagus nerve and were injected i.p. with either saline or paraquat and sacrificed at 2 days after treatment. (A) Representative images show left $\mathrm{DMnX}$ tissue stained with anti-nitrated $\alpha$-synuclein (nSyn24). Squares highlight neuritic immunoreactivity, while the arrowhead indicates a DMnX immunoreactive cell body. Scale bar: $10 \mu \mathrm{m}$. (B) Confocal images show a neuron immunoreactive for both nSyn24 and h $\alpha$-synuclein in the left DMnX of a mouse treated with h $\alpha$-synuclein AAVs/paraquat. Scale bar: $5 \mu \mathrm{m}$. (C) Representative low- and high-magnification images of the left DMnX (delineated in orange) show specific signal for nitrated h $\alpha$-synuclein detected by indirect h $\alpha$-synuclein/3-NT PLA. CC, central canal. Scale bars: $200 \mu \mathrm{m}$ (left); $10 \mu \mathrm{m}$ (right). (D) The number of PLA dots was counted in the left DMnX of mice treated with h $\alpha$-synuclein AAVs/saline ( $n=4$, gray bar) or h $\alpha$-synuclein AAVs/paraquat ( $n=4$, red bar). (E and F) Sections of the medulla oblongata were double labeled with PLA and anti-h $\alpha$-synuclein. Representative images show cell bodies (E) and neurites (F) in the left DMnX. Scale bars: $5 \mu \mathrm{m}$. (G) Nitrated $\alpha$-synuclein was measured in the dorsal left medulla oblongata by ELISA. OD was compared in samples from mice treated with h $\alpha$-synuclein AAVs/saline $(n=9)$ versus h $\alpha$-synuclein AAVs/paraquat $(n=9)$. (H and $\mathbf{I})$ Medullary sections were double stained with Syn 505 and anti-ho-synuclein. (H) Representative confocal images show left DMnX tissue. Scale bar: $10 \mu m$. (I) Fluorescence densities for Syn 505 and ho-synuclein were measured on images of the left DMnX of mice treated with h $\alpha$-synuclein AAVs/saline ( $n=4$ ) or h $\alpha$-synuclein AAVs/ paraquat $(n=5)$. At least 6 images/animal were analyzed. Values were calculated as the ratio of Syn 505/h $\alpha$-synuclein fluorescence and are expressed as percentage of the mean value in h $\alpha$-synuclein AAV/saline-injected animals. Box and whisker plots are shown. ${ }^{*} P \leq 0.05$, Mann-Whitney $U$ test.

more robust DHE oxidation within cell bodies and neurites in the left DMnX, as assessed histologically as well as by quantification of the perikaryal fluorescent signal (Figure 2, B and C). The more severe oxidative stress caused by combined ho-synuclein overexpression and paraquat challenge was associated with enhanced neurotoxicity. Overexpression of h $\alpha$-synuclein followed by 2 saline injections induced a 5\% decrease in the number of DMnX neurons at both 2 and 7 days after treatment (Figure 2D). In contrast, cotreatment with ho-synuclein AAVs and paraquat caused a
$15 \%$ reduction of DMnX Nissl-stained neurons already at 2 days after the second paraquat injection; this reduction progressed to a $25 \%$ loss at the 7-day time point (Figure 2D).

Oxidative modifications of $\alpha$-synuclein in the DMnX. Oxidative $\alpha$-synuclein modifications are typically observed in human synucleinopathies. Antibodies that specifically detect nitrated $\alpha$-synuclein or react against 3-nitrotyrosine-modified (3-NTmodified) protein residues robustly label $\alpha$-synuclein-containing inclusions in the brain of patients with PD and other synucle- 
A
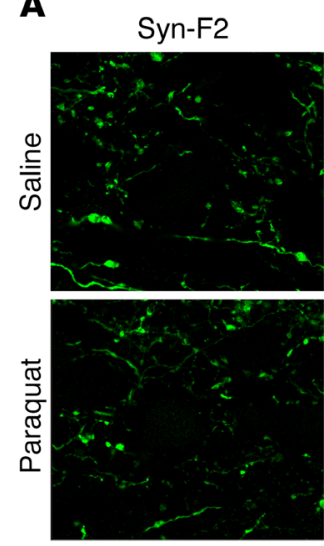

C
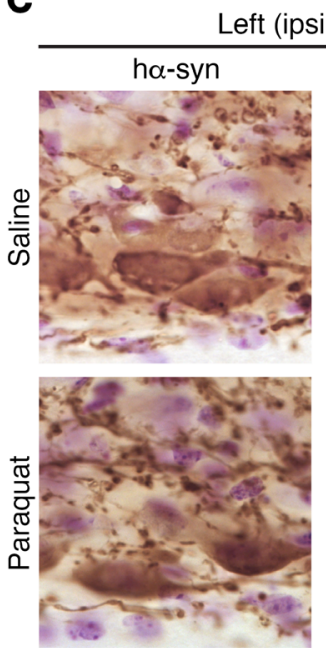

ha-syn
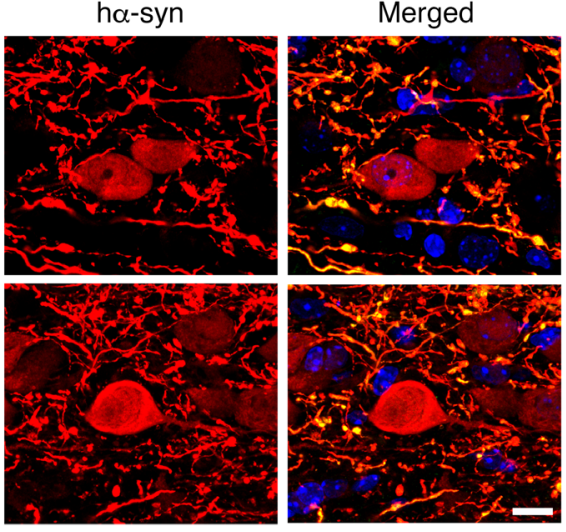

B

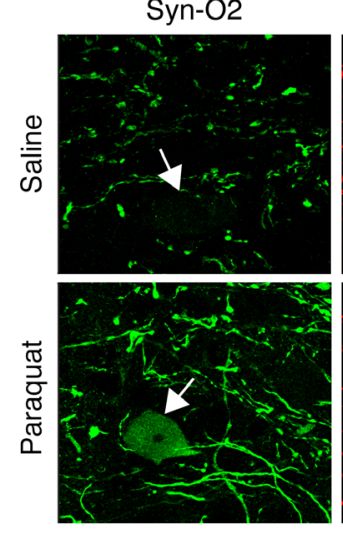

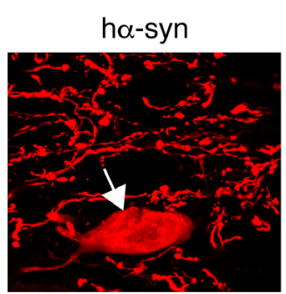

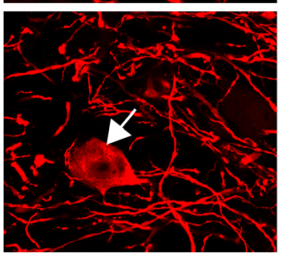

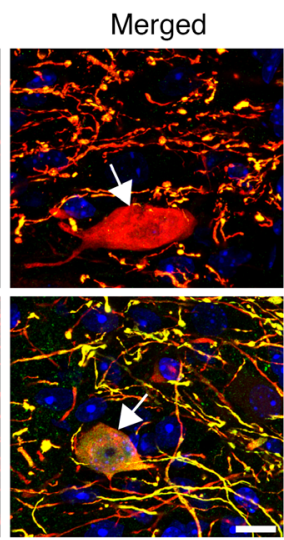

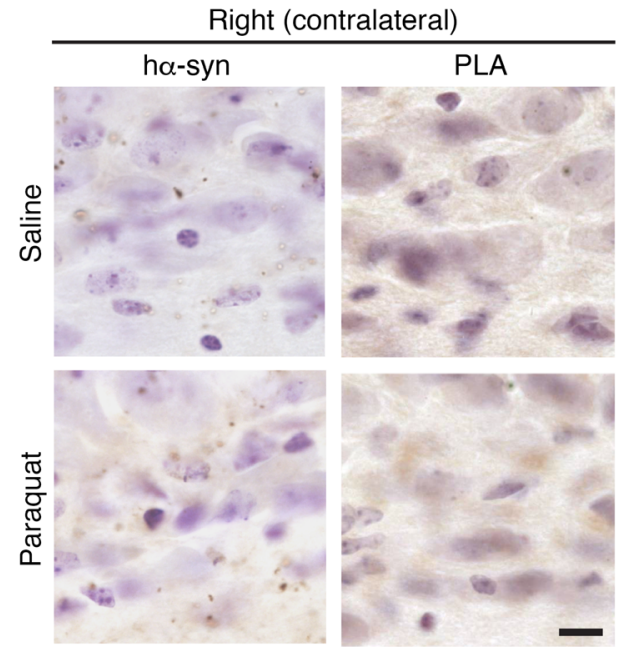

\section{D \\ D}

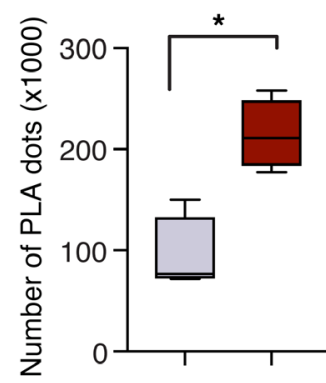

AAV/saline

AAV/paraquat

Figure 4. Severity of oxidative stress affects the extent of $\alpha$-synuclein aggregation. Mice received an injection of ho-synuclein-carrying AAVs into the left vagus nerve and were injected systemically with either saline or paraquat and sacrificed at 2 days after treatment. (A) Representative confocal images show left DMnX tissue double labeled with an antibody that recognizes only mature $\alpha$-synuclein fibrils (Syn-F2) and with anti-h $\alpha$-synuclein. Scale bar: $5 \mu \mathrm{m}$. (B) Representative confocal images of left DMnX tissue. One of the antibodies used for double staining detects oligomeric and fibrillar forms of $\alpha$-synuclein (Syn-02). The other antibody was anti-h $\alpha$-synuclein. Arrows indicate 2 h $\alpha$-synuclein-positive neurons, one from a mouse injected with saline and the other from an animal treated with paraquat; the latter is also Syn-02 immunoreactive. Scale bar: $5 \mu$ m. (C) Sections of the medulla oblongata were labeled with anti-h $\alpha$-synuclein or with syn/syn PLA, which detects aggregated (preferentially oligomeric) h $\alpha$-synuclein. Protein overexpression and aggregation were visualized in the left (ipsilateral to AAV infusion) and right (contralateral) DMnX and compared in mice treated with h $\alpha$-synuclein AAVs/ saline versus h $\alpha$-synuclein AAVs/paraquat. Scale bars: $10 \mu \mathrm{m}$. (D) The number of syn/syn PLA dots was counted stereologically in the left DMnX from mice treated with h $\alpha$-synuclein AAVs/saline ( $n=4$, gray bar) or with h $\alpha$-synuclein AAVs/paraquat $(n=4$, red bar). Box and whisker plots show median, upper and lower quartiles, and maximum and minimum as whiskers. ${ }^{*} P \leq 0.05$, Mann-Whitney $U$ test.

inopathies $(26,27)$. Furthermore, monoclonal antibodies (i.e., Syn 505, Syn 506, Syn 514, and Syn 303) that are raised against oxidized/nitrated recombinant ho-synuclein recognize conformational $\alpha$-synuclein variants that are preferentially associated with PD pathological lesions $(28,29)$. Here, the relationship between ROS formation and $\alpha$-synuclein nitration was investigated in the DMnX of AAV-injected animals and compared under conditions of milder (AAV/saline treatment) versus severe (AAV/paraquat treatment) oxidative stress. Mice were sacrificed at 2 days after the second saline or paraquat treatment, and tissue sections of the medulla oblongata were immunostained with mouse antinitrated $\alpha$-synuclein clone 24.8 (nSyn24), an antibody that detects $\alpha$-synuclein nitrated at positions Y125 and Y133 (26). NSyn24 labeling was sparse in the left (AAV-injected side) DMnX from saline-treated animals and revealed a neuritic, dot-like accumu- lation of the modified protein (Figure 3A). More robust staining characterized the DMnX of paraquat-injected mice in which nSyn24 immunoreactivity not only labeled neuritic projections, but could also be detected within neuronal cell bodies overexpressing ho-synuclein (Figure 3, A and B). To further evaluate and quantify $\alpha$-synuclein nitration, fixed medullary tissue sections and homogenates from snap-frozen medulla oblongata specimens were processed for proximity ligation assay (PLA) and ELISA, respectively. PLA has previously been shown to enable visualization of proteins with specific posttranslational modifications, such as phosphorylation, SUMOylation, and acetylation (30-32). In the present study, a pair of antibodies, namely anti-3-NT and MJFR1, was used to detect nitrated ho-synuclein by direct or indirect PLA; the former involved direct conjugation of the primary antibodies to oligonucleotide probes, whereas for the latter, reactive oligo- 
A
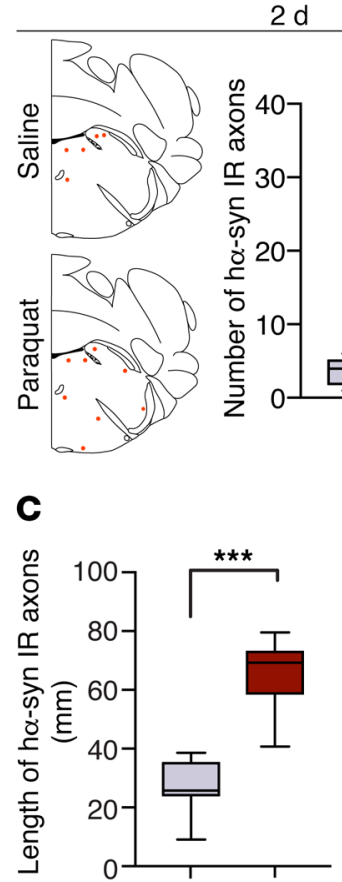

$2 d$

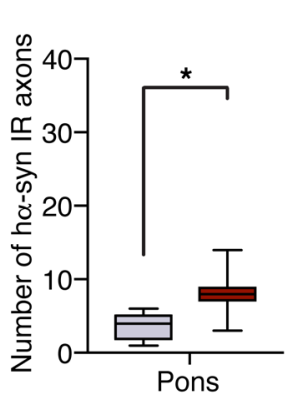

B

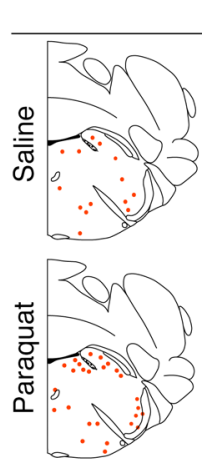

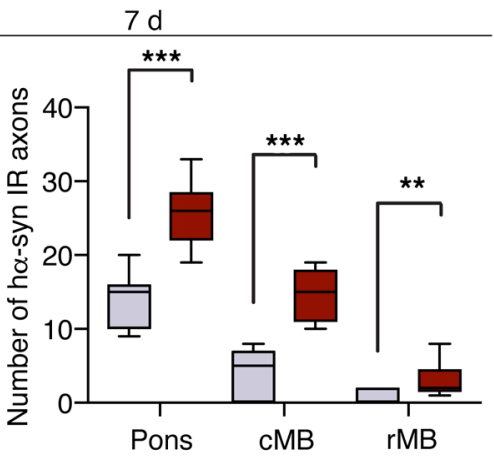

E

D
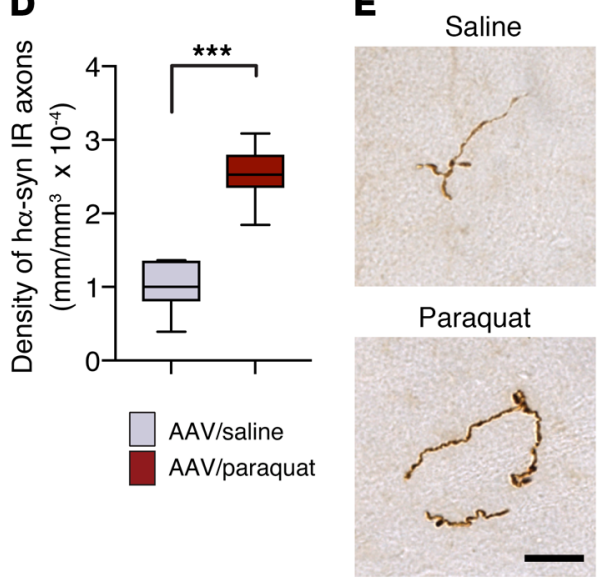

F Saline

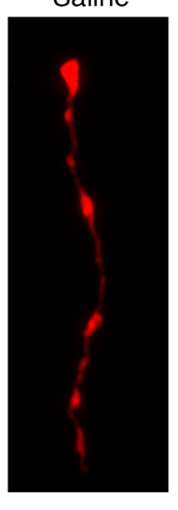

Paraquat

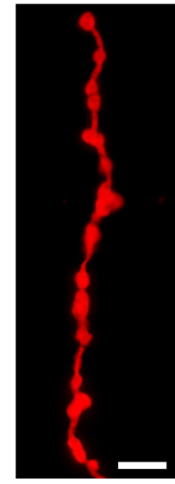

Figure 5. Oxidative stress promotes caudo-rostral spreading of ho-synuclein in the mouse brain. Mice received an infusion of h $\alpha$-synuclein-carrying AAVs into the left vagus nerve and were injected i.p. with either saline or paraquat and sacrificed at 2 or 7 days after treatment. Tissue sections were immunostained with anti-h $\alpha$-synuclein. (A and B) The schematic plots show the distribution of ho-synuclein-labeled axons (each red dot represents one of these axons) in the left (AAV-injected side) pons. In the graphs, data show the counts of h $\alpha$-synuclein-immunoreactive axons in the left pons at 2 and 7 days and in the caudal (cMB) and rostral midbrain (rMB) at 7 days. Tissue was obtained from mice treated with ho-synuclein AAVs/saline $(n=6$ at 2 days and $n=7$ at 7 days, gray bars) or with h $\alpha$-synuclein AAVs/paraquat ( $n=7$ at 2 days and $n=9$ at 7 days, red bars). (C and D) Length and density of h $\alpha$-synuclein-positive axons were estimated at 7 days after treatment in a pontine area encompassing the locus coeruleus and the nucleus parabrachialis using the Spaceballs stereological tool. Analyses were made in the left pons in samples collected from mice treated with h $\alpha$-synuclein AAVs/saline $(n=7)$ or with h $\alpha$-synuclein AAVs/paraquat $(n=9)$. (E and $\mathbf{F})$ Representative images of pontine axons immunolabeled with anti-h $\alpha$-synuclein and visualized using brightfield (brown) or fluorescent (red) microscopy. Scale bars: $20 \mu \mathrm{m}(\mathbf{E}) ; 5 \mu \mathrm{m}$ (F). Box and whisker plots show median, upper and lower quartiles, and maximum and minimum as whiskers. ${ }^{*} P \leq 0.05 ;{ }^{* *} P \leq 0.01 ;{ }^{* *} P \leq 0.001$, Mann-Whitney $U$ test.

nucleotides were conjugated to the secondary antibodies. Both ho-synuclein/3-NT PLA procedures yielded similar findings. Specific chromogenic dots were detected in the left but not the right DMnX of all AAV-injected mice, and a marked enhancement of signal characterized sections from paraquat- as compared with saline-treated animals (Figure 3C and Supplemental Figure 3). This effect was confirmed and quantified by PLA dot counts (Figure 3D). Furthermore, using an in situ fluorescent PLA protocol, neuronal cell bodies and neuritic projections accumulating nitrated ha-synuclein were frequently observed in the DMnX of mice injected with paraquat and were instead very scarce in saline-treated animals (Figure 3, E and F). Levels of nitrated $\alpha$-synuclein were finally measured by ELISA in tissue specimens of the dorsal left medulla oblongata encompassing the transduced DMnX. Using an antibody against total (human and mouse) $\alpha$-synuclein as capture reagent and anti-3-NT as a detection antibody, we found protein nitration to be significantly increased as a consequence of paraquat administration (Figure $3 \mathrm{G}$ ).
Medullary tissue sections were then double-immunostained with Syn 505, one of the antibodies raised against oxidized/ nitrated ho-synuclein (28), and anti-h $\alpha$-synuclein. Results showed immunoreactivity within transduced cell bodies and neurites in the left DMnX of saline-injected mice. The extent of protein modifications detected by Syn 505 was more pronounced, however, in animals treated with paraquat (Figure $3 \mathrm{H}$ ). Similarly, when the ratio of fluorescent Syn 505/ho-synuclein signals was measured in DMnX sections, a significant increase was found after paraquat administration (Figure 3I).

Oxidative stress-induced $\alpha$-synuclein aggregation in the DMnX. Oxidative stress may exacerbate $\alpha$-synuclein's tendency to aggregate (33-36). Furthermore, nitrative modifications of $\alpha$-synuclein induced by oxidative stress could affect the kinetics of protein aggregation and modulate pathways of protein assembly, leading to the formation of oligomeric and/or fibrillar species (3740). Conformation-specific antibodies were used to determine whether oxidative stress enhanced $\alpha$-synuclein aggregation in 
A

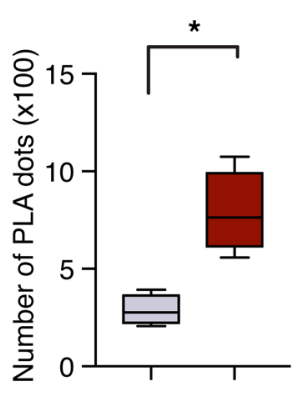

B
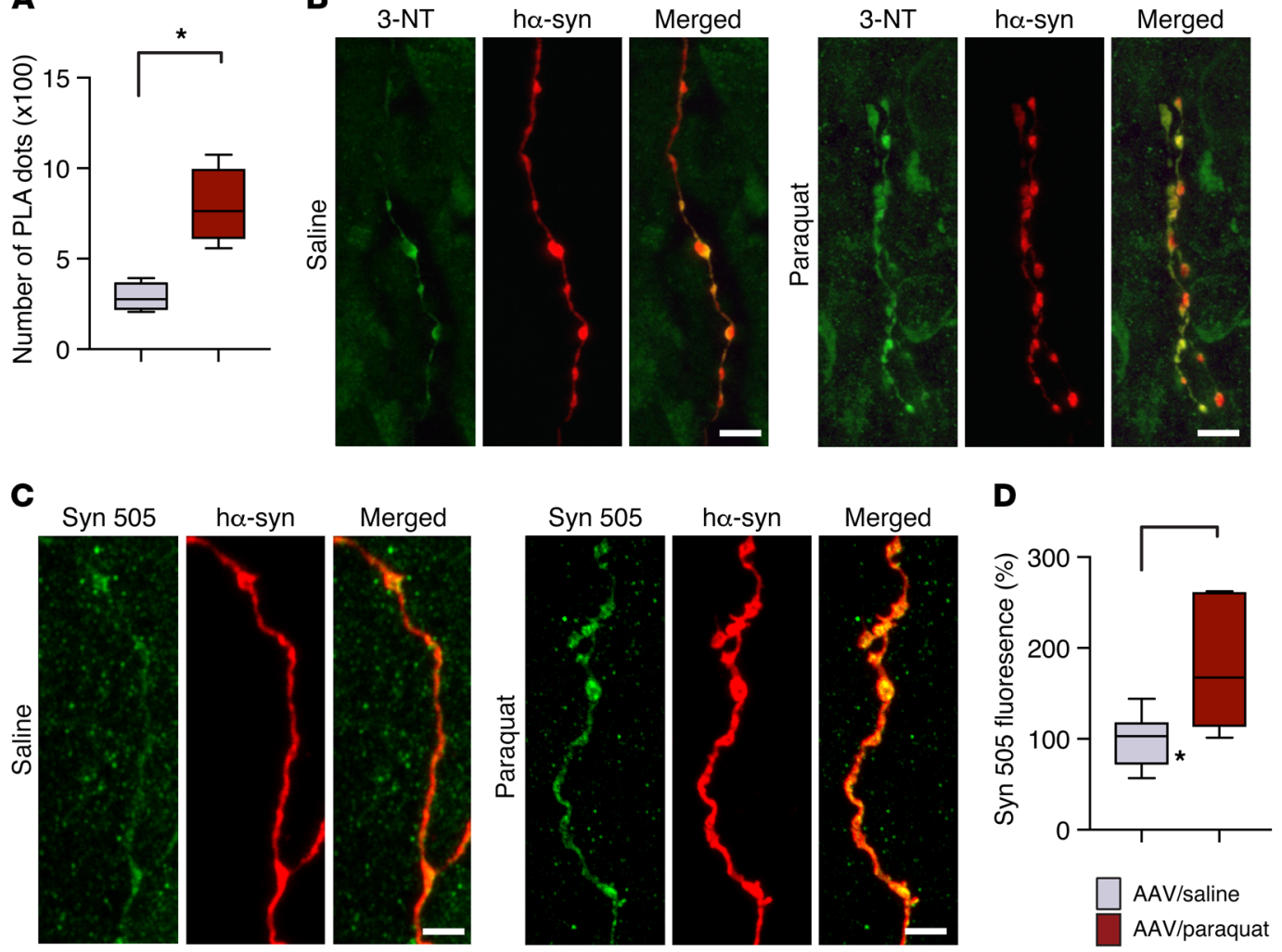

Figure 6. Oxidatively modified ho-synuclein is accumulated within pontine axons during caudo-rostral spreading of the protein. Mice received an infusion of h $\alpha$-synuclein-carrying AAVs into the left vagus nerve and were injected systemically with either saline or paraquat and sacrificed at 7 days after treatment. (A) Nitrated h $\alpha$-synuclein was detected by indirect h $\alpha$-synuclein/3-NT PLA. The number of PLA dots in the left pons from mice treated with h $\alpha$-synuclein AAVs/saline ( $n=4$, gray bar) or with h $\alpha$-synuclein AAVs/paraquat ( $n=5$, red bar) was counted. (B) Pontine tissue sections were costained with anti-3-NT and anti-h $\alpha$-synuclein. Representative confocal images show labeled axons in the left pons. Scale bars: $5 \mu$ m. (C) Representative confocal images show axons in the left pons stained with anti-Syn 505 and anti-h $\alpha$-synuclein. Scale bar: $5 \mu \mathrm{m}$. (D) Measurements of Syn 505 fluorescence were carried out in the left pons of mice treated with h $\alpha$-synuclein AAVs/saline $(n=7)$ or with h $\alpha$-synuclein AAVs/paraquat ( $n=6)$. At least 3 axons/animal were analyzed and averaged. Values are expressed as percentage of the mean value in ho-synuclein AAV/saline-injected animals. Box and whisker plots show median, upper and lower quartiles, and maximum and minimum as whiskers. ${ }^{*} P \leq 0.05$, Mann-Whitney $U$ test.

the $\mathrm{DMnX}$ and whether it promoted the accumulation of specific aggregate forms (i.e., fibrillar or oligomeric). First, medullary tissue sections from ho-synuclein-overexpressing mice were stained with an antibody, Syn-F2, that recognizes only mature amyloid $\alpha$-synuclein fibrils $(20,41)$. Immunoreactivity for Syn-F2 labeled h $\alpha$-synuclein-containing neuronal processes in the left DMnX, with no apparent difference in the distribution and robustness of labeling between overexpressing mice injected with saline and overexpressing animals treated with paraquat (Figure 4A). Tissue sections were then probed with a second antibody, Syn-O2, capable of detecting both $\alpha$-synuclein oligomers and $\alpha$-synuclein fibrils $(20,41)$. Syn-O2 staining was overtly more pronounced in specimens from paraquat- as compared with saline-injected mice, as indicated by a denser network of labeled neurites and by the presence of DMnX cell bodies immunoreactive for both Syn-O2 and ho-synuclein (Figure 4B). Taken together, these findings indicate that, as a result of paraquat-induced oxidative stress, $\alpha$-synuclein aggregation is enhanced and leads to the formation of predominantly oligomeric protein species. Similar conclusions could be drawn from experiments in which protein aggregation was assessed using a PLA protocol (syn/syn PLA) that preferen- tially detects pathological h $\alpha$-synuclein oligomers in PD brain and in the brain of ha-synuclein-overexpressing mice $(20,42)$. Syn/syn PLA labeling was observed in medullary sections from all AAV-injected mice, and its distribution closely matched the pattern of ho-synuclein overexpression. In particular, distinct chromogenic spots characterized the left DMnX (ipsilateral to the AAV injection side), whereas no specific signal was detected on the contralateral side of the brain (Figure 4C). PLA reactivity was then compared between overexpressing mice treated with saline versus paraquat; results in this latter group of animals showed enhanced tissue labeling, a significant increase in PLA dot counts, and a more pronounced accumulation of PLA-labeled $\alpha$-synuclein oligomers within DMnX-overexpressing cell bodies and neurites (Figure 4, C and D, and Supplemental Figure 4).

Oxidative stress and neuron-to-neuron $\alpha$-synuclein transfer. Overexpression of h $\alpha$-synuclein in the rodent DMnX, as induced by vagal AAV injections, has been shown to trigger a discrete, progressive accumulation of the exogenous protein within dystrophic axons in the pons, midbrain, and forebrain. Since DMnX cholinergic neurons do not have central axons projecting toward higher brain regions, this observation of extramedullary pathol- 
A
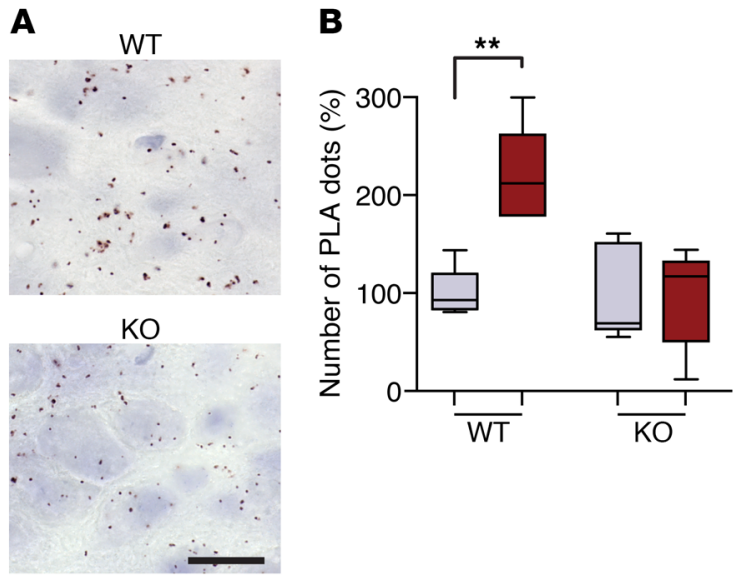

C

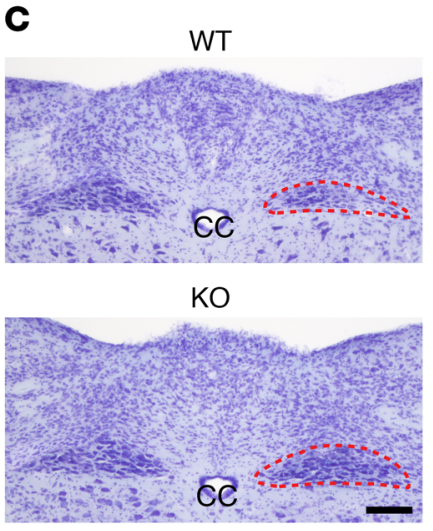

E

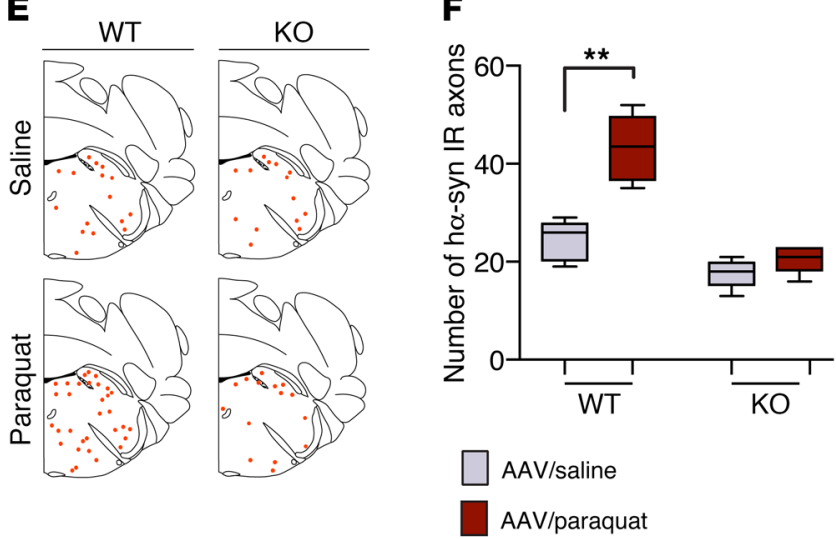

ogy reflects an interneuronal transfer and subsequent retrograde spreading of $h \alpha$-synuclein $(20,25,43)$. The next set of experiments was designed to investigate whether vulnerability of the DMnX to oxidative stress may affect neuron-to-neuron $\alpha$-synuclein transmission in this model of caudorostral protein spreading. Tissue sections of the left pons and midbrain were collected from mice that first received h $\alpha$-synuclein AAVs and were then treated with 2 injections of saline or paraquat. Samples were stained with a ho-synuclein-specific antibody (Syn211) and analyzed for the presence of the exogenous protein. A few axonal projections immunoreactive for h $\alpha$-synuclein were already present in pontine sections at 2 days after the second saline/paraquat administration, and interestingly, counts of these labeled axons revealed a higher
Figure 7. Gp91phox-deficient mice are resistant to paraquat-induced DMnX pathology. WT and gp91phox-deficient (KO) mice received an injection of h $\alpha$-synuclein AAVs into the left vagus nerve and were injected systemically with either saline ( $n \geq 4$ /genotype, gray bars) or paraquat ( $n \geq 4$ /genotype, red bars) and sacrificed at 7 days. (A) Medullary tissue sections were processed for indirect h $\alpha$-synuclein/3-NT PLA. Representative images show the left $\mathrm{DMnX}$ of a WT and a KO mouse injected with h $\alpha$-synuclein AAVs/paraquat. Specific PLA signal was apparently reduced in the KO mouse. Scale bar: $10 \mu \mathrm{m}$. (B) The number of h $\alpha$-synuclein/3-NT PLA dots was counted in the left DMnX of WT and KO mice injected with h $\alpha$-synuclein AAVs/saline or h $\alpha$-synuclein AAVs/paraquat. Values are expressed as percentage of the mean value in the corresponding h $\alpha$ synuclein AAV/saline-injected animals. (C) Representative images of Nissl-stained neurons in the dorsal medulla oblongata. Tissues were obtained from a WT and a KO mouse injected with ha-synuclein AAVs/ paraquat. The left $D M n X$ is delineated in red. Neuronal loss is evident only in the left (ipsilateral to AAV infusion) DMnX from the WT animal. Scale bar: $100 \mu \mathrm{m}$. (D) The number of Nissl-stained neurons was counted stereologically in the left DMnX of WT and KO mice treated with h $\alpha$-synuclein AAVs/saline or h $\alpha$-synuclein AAVs/paraquat. Control counts ( $n=5$, light blue bar) were obtained from the right DMnX of WT animals injected with h $\alpha$-synuclein AAVs/saline. (E and $\mathbf{F}$ ) Pontine tissue sections were immunostained with anti-h $\alpha$-synuclein. The schematic plots show the distribution of h $\alpha$-synuclein-labeled axons in the left pons. In the graph, data show the counts of h $\alpha$-synuclein-positive axons in the left pons. Box and whisker plots show median, upper and lower quartiles, and maximum and minimum as whiskers. ${ }^{* *} P \leq 0.01$; ${ }^{* *} P \leq 0.001$, Mann-Whitney $U$ test (saline- versus paraquat-injected mice) or KruskalWallis followed by Dunn's post hoc (D).

number in paraquat- as compared with saline-treated animals (Figure 5A). Additional measurements were carried out at a later time point, namely 7 days after saline/paraquat treatment. The number of ha-synuclein-positive axons was increased in pontine sections at 7 days, and at this later time point, immunoreactive fibers were also detected in the caudal and rostral midbrain. In findings similar to those at 2 days, counts of axons containing h $\alpha$-synuclein were consistently higher in pontine and midbrain sections from mice injected with paraquat (Figure 5B). Length and density of labeled fibers were then measured at the 7-day time point in a defined pontine region encompassing the locus coeruleus and parabrachial nucleus $(20,44)$. Both measurements showed values that were more than doubled as a result of paraquat administration (Figure 5, C and D). Axons accumulating h $\alpha$-synuclein generally appeared enlarged, tortuous, and with irregularly spaced varicosities. When fiber morphology was compared in sections from saline- versus paraquat-treated mice, these features were noticeably more prominent in the latter group of animals. In particular, axons from the paraquat-exposed group were more swollen, had larger varicosities, and displayed stronger diaminobenzidine and fluorescent labeling, all consistent with an enhanced h $\alpha$-synuclein load (Figure 5, E and F).

Further characterization of the spreading protein involved detection and quantification of nitrated and Syn 505-immunoreactive h $\alpha$-synuclein in pontine tissue. Specific h $\alpha$-synuclein/ 3-NT PLA labeling (indirect PLA) was very scant in samples from saline-treated mice, but became more evident and abundant after paraquat administration (Figure 6A). Similarly, double staining of pontine sections with anti-3-NT and anti-hosynuclein revealed fibers with more pronounced colocalization in paraquat-injected mice (Figure 6B). Severe oxidative stress and 
A
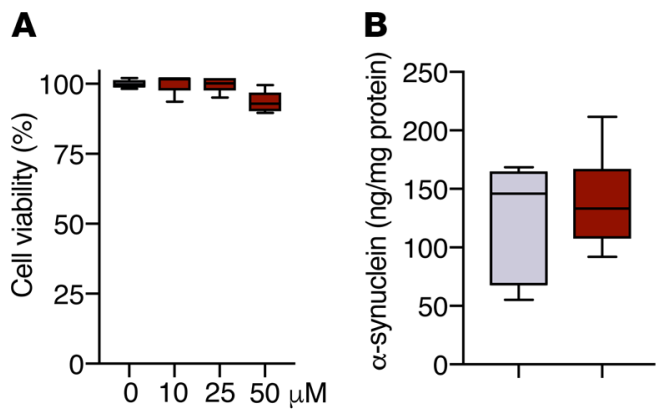

C

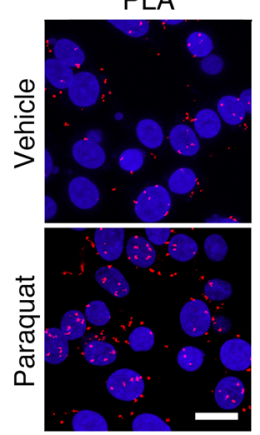

D

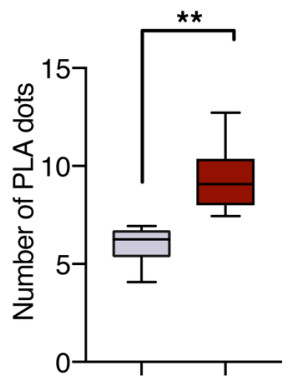

$\mathbf{E}$

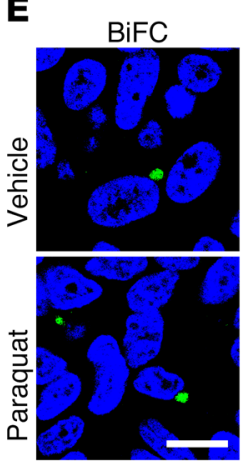

$\mathbf{F}$

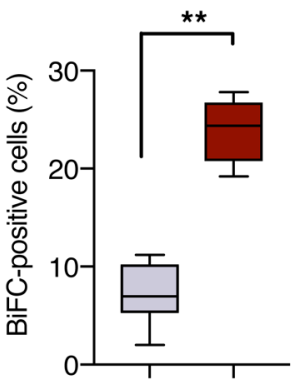

H
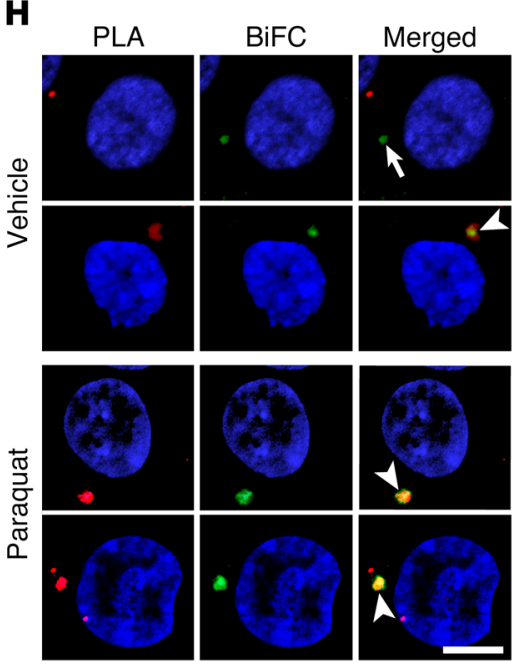

J Vehicle

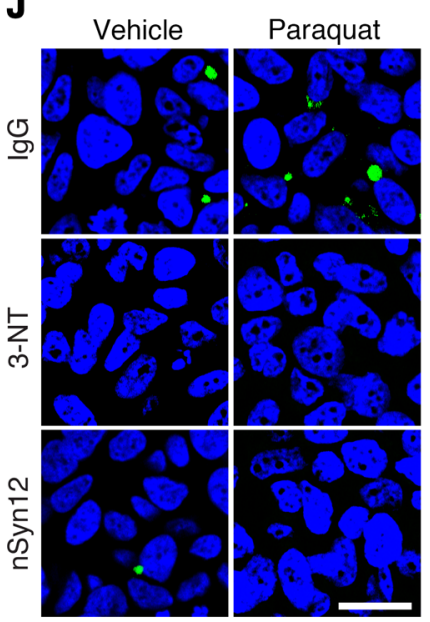

$\mathbf{K}$
G

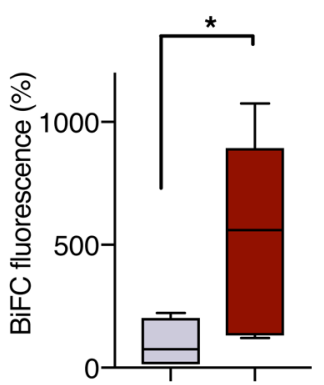

Figure 8. Cell-to-cell ha-synuclein exchange is promoted by oxidative stress in vitro. (A) Cocultures of V1S- and SV2expressing cells were incubated for 2 days with vehicle ( $n=6$ separate experiments, gray bar) or different concentrations of paraquat ( $n=6 /$ concentration, red bars). Cell viability was measured and expressed as percentage of vehicle-treated cultures. (B) Levels of h $\alpha$-synuclein were measured in cocultures treated with vehicle $(n=6)$ or $25 \mu \mathrm{M}$ paraquat $(n=6)$ by ELISA. (C and $\mathbf{D})$ Representative images show accumulation of nitrated h $\alpha$-synuclein in the form of h $\alpha$-synuclein/3-NT PLA dots (red). Cell nuclei were stained with DAPI (blue). Scale bar: $10 \mu \mathrm{m}$. The number of PLA dots was counted in cultures treated with vehicle $(n=4)$ or paraquat $(n=4)$. A minimum of 100 cells/experiment were analyzed. PLA counts were divided by the number of cells, and values were averaged. (E) Representative images show BiFC (green) as a marker of h $\alpha$-synuclein transfer into recipient cells. Scale bar: $20 \mu \mathrm{m}$. (F and $\mathbf{G})$ The percentage of BiFC-positive cells ( $n=6 /$ treatment, $\mathbf{F}$ ) and cell fluorescence intensity ( $n=6$ /treatment, $\mathbf{C}$ ) were compared in cultures treated with vehicle or paraquat. Integrated density of $\mathrm{BiFC}$ fluorescence was measured in a minimum of 400 cells/ experiment and expressed as percentage of the mean value in vehicle-treated cultures. (H and I) Representative images show h $\alpha$-synuclein/3-NT PLA (red) and BiFC (green) fluorescence. The arrow indicates lack of signal colocalization, while the arrowheads show colocalization. Scale bar: $5 \mu \mathrm{m}$. The percentage of BiFC aggregates colocalizing with PLA was calculated in cultures treated with vehicle $(n=4)$ or paraquat $(n=4)$. Minimum 100 cells/experiment. (J and $\mathbf{K}$ ) Representative images show BiFC (green) in cocultures treated with saline or paraquat in the presence of IgG, anti-3-NT, or anti-nitrated $\alpha$-synuclein (nSyn12). Scale bar: $20 \mu \mathrm{m}$. The percentage of BiFC-positive cells ( $n=6$ /treatment) was calculated under different treatment conditions. ${ }^{*} P \leq 0.05 ;{ }^{* *} P \leq 0.01$, Mann-Whitney $U$ test or Kruskal-Wallis followed by Dunn's post hoc test (K).

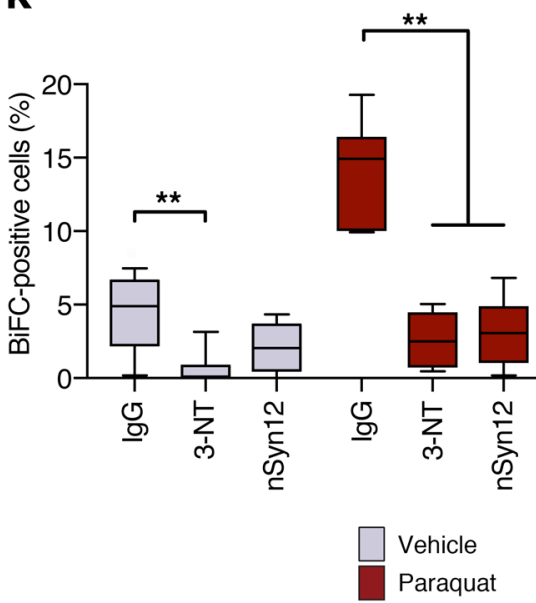


increased spreading induced by the AAV/paraquat treatment was also associated with enhanced accumulation of Syn 505-reactive h $\alpha$-synuclein species within pontine fibers; after labeling with anti-Syn 505, the fluorescent signal within these fibers was almost doubled in sections from AAV/paraquat- compared with AAV/ saline-treated animals (Figure 6, C and D).

Prevention of paraquat-induced DMnX pathology. A likely mechanism contributing to ROS production after paraquat exposure involves paraquat-microglia interactions. More specifically, microglial membrane-bound NADPH oxidase could catalyze the 1-electron reduction of paraquat, thus promoting its redox cycling with molecular oxygen and enhancing the formation of superoxide and other ROS $(45,46)$. To assess this mechanism and further evaluate the contribution of ROS production to DMnX pathology, experiments were carried out in mutant mice lacking the gp91phox subunit of NADPH oxidase. Absence of this subunit prevents assembly of the enzyme at the plasma membrane and thus abolishes its catalytic activity $(45,47)$. Gp91phoxdeficient mice on the C57BL/6J background strain and C57BL/ 6J WT animals were administered h $\alpha$-synuclein AAVs intravagally and then injected twice with saline or paraquat. They were sacrificed at 7 days after the second saline/paraquat treatment. To evaluate oxidative/nitrative stress, sections of the medulla oblongata were processed for indirect h $\alpha$-synuclein/3-NT PLA, and the number of PLA dots was compared in the DMnX of WT and mutant mice after saline or paraquat administration (Figure 7, A and B). Stereological counts of DMnX neurons were carried out to compare the neurodegenerative effects of paraquat administration in WT verus mutant mice (Figure 7, C and D). Finally, the number of neuronal fibers immunoreactive for ho-synuclein was counted in the pons as a measure of neuron-to-neuron protein transfer and retrograde axonal spreading (Figure 7, E and F). Results showed that, as seen in the earlier experiments, paraquat administration caused an enhancement of ho-synuclein nitration, DMnX neuronal loss, and ho-synuclein spreading in WT animals. Quite remarkably, these effects were abolished in gp91phox-deficient mice. In these animals, the counts of DMnX PLA dots, DMnX neurons, and pontine h $\alpha$-synuclein-containing axons were comparable regardless of treatment with $\mathrm{AAV} /$ saline or $\mathrm{AAV} /$ paraquat (Figure 7, B, D, and F).

Oxidative stress and cell-to-cell $\alpha$-synuclein transfer in vitro. Results in vivo indicating enhanced ho-synuclein spreading in paraquat-treated mice are consistent with the interpretation that paraquat-induced oxidative stress promotes $\alpha$-synuclein transfer from donor to recipient neurons. Cell-to-cell $\alpha$-synuclein transmission in the absence or presence of oxidative stress was further investigated next using an in vitro system in which 2 separate $\mathrm{SH}-\mathrm{SY} 5 \mathrm{Y}$-derived cell lines are cocultured; the 2 cell lines stably express h $\alpha$-synuclein fused with either the N (V1S) or C terminal (SV2) fragment of the fluorescent protein Venus. In this system, bimolecular fluorescence complementation (BiFC) indicates dimerization or oligomerization of h $\alpha$-synuclein as a consequence of its transmission and exchange between V1S- and SV2-containing cells (48). In a set of initial experiments, V1S- and SV2-expressing cells were cocultured in the presence of vehicle or different concentrations of paraquat. Measurements of cell viability determined that no toxicity occurred after a 48-hour incubation with 10 or $25 \mu \mathrm{M}$ paraquat; with $50 \mu \mathrm{M}$ paraquat, a $10 \%$ loss of viability occurred, although this effect did not reach statistical significance (Figure 8A). Based on these findings, all subsequent experiments were carried out on cells treated with vehicle or $25 \mu \mathrm{M}$ paraquat for 48 hours. Under these conditions, treatment with paraquat did not significantly change cellular levels of h $\alpha$-synuclein as assessed by ELISA (Figure $8 \mathrm{~B}$ ). To assess the oxidative effect of paraquat, nitration of intracellular h $\alpha$-synuclein was visualized and quantified with h $\alpha$-synuclein/ 3-NT PLA (indirect PLA). Nitrated ha-synuclein was detected within control cells incubated in the presence of vehicle; however, a significantly higher count of PLA dots characterized cells in paraquat-exposed cultures (Figure 8, C and D). Cell-to-cell h $\alpha$-synuclein transfer was assessed by counting the number of cells with BiFC-positive structures (48). Furthermore, total cell fluorescence caused by bimolecular complementation was measured and compared in randomly selected cells from vehicleversus paraquat-treated cultures. Both measurements revealed a significant increase in BiFC caused by paraquat, supporting an association between paraquat-induced oxidative stress and enhanced ho-synuclein transmission (Figure 8, E-G). Further evidence of this association derived from experiments in which cocultures of V1S- and SV2-expressing cells were challenged with oxidative stress-inducing agents other than paraquat. In particular, the percentage of BiFC-positive cells was found to be more than doubled in cultures treated with hydrogen peroxide versus vehicle; it was also increased by approximately 5 times over control levels when hydrogen peroxide was added together with iron sulfate to the incubation medium (Supplemental Figure 5).

Coaggregates of V1S and SV2 proteins formed as a consequence of bimolecular complementation contained nitrated h $\alpha$-synuclein, as indicated by colocalization of fluorescent dots generated by h $\alpha$-synuclein/3-NT PLA within BiFC-positive structures (Figure $8 \mathrm{H}$ ). h $\alpha$-Synuclein/3-NT PLA colabeled approximately $60 \%$ of BiFC-positive aggregates in vehicle-treated cultures, whereas almost $100 \%$ colocalization of PLA and BiFC was found in cells exposed to paraquat (Figure 8I). These findings raise the possibility that nitrated forms of h $\alpha$-synuclein may play an important role in protein transmission, particularly under conditions of oxidative stress. Cell-to-cell transfer of nitrated h $\alpha$-synuclein would likely involve its initial release into the extracellular space. A final set of experiments was therefore designed to test the hypothesis that addition of antibodies capable of binding extracellular nitrated $h \alpha$-synuclein may counteract protein transfer and formation of BiFC-positive oligomers in this in vitro system. Two specific antibodies were used for these experiments, namely anti-3-NT or anti-nitrated $\alpha$-synuclein clone nSyn12. A control antibody, mouse IgG, was also used to account for nonspecific protein binding. IgG, anti-3-NT, or nSyn12 was added to cultures treated with either vehicle or paraquat. In vehicle-treated cultures, a comparison of the percentage of cells containing BiFCpositive aggregates after addition of IgG versus anti-3-NT or nSyn12 revealed decreased fluorescence in the presence of specific nitrated protein-binding antibodies; this decrease reached statistical significance with anti-3-NT, but not nSyn12, however (Figure 8, J and K). In paraquat-treated cultures, the effects of the 2 specific antibodies were both statistically significant $(P \leq 0.01)$. 
Addition of either anti-3-NT or nSyn12 largely prevented the increase in ho-synuclein transfer caused by paraquat and reduced cellular formation of BiFC-positive aggregates by approximately $80 \%$ (Figure 8, J and K).

\section{Discussion}

Oxidative challenges are likely to be common events in the CNS, underscoring the relevance of oxidative stress for pathogenetic processes in human neurodegenerative diseases. They can result from cellular dysfunction, such as mitochondrial impairment, and tissue reactions, such as activation of innate immunity (49-51). In $\mathrm{PD}$, oxidative stress could also be promoted by disease risk factors, including aging, environmental exposures, and genetic variants $(22,49)$. Here, we demonstrate a high susceptibility of DMnX neurons to oxidative stress; our data also indicate that these neurons are relatively more vulnerable to oxidative stress than other populations of cholinergic cells. Following exposure to paraquat, DMnX neurons accumulated substantial amounts of ROS, whereas under the same toxic conditions, no apparent ROS buildup was detected within cholinergic cells in the nearby hypoglossal nucleus nor in the striatum and medial septal nucleus.

This differential vulnerability, in spite of the same neurotransmitter phenotype, is not unique to cholinergic neurons, but is instead reminiscent of differences seen between midbrain dopaminergic cells. Dopaminergic neurons in the substantia nigra pars compacta are markedly more susceptible to oxidative challenges and ROS accumulation than dopaminergic cells in the adjacent ventral tegmental area $(3,52,53)$. The substantia nigra pars compacta is also known to be more vulnerable than the ventral tegmental area to the neurodegenerative process of PD $(54,55)$. Similarly, earlier and more prominent PD pathology, in the form of both $\alpha$-synuclein lesions and neurodegeneration, characterizes the DMnX and distinguishes DMnX neurons from other cholinergic cells in the hypoglossal nucleus, striatum, and medial septum $(10,56-58)$. Thus, taken together, previous and current observations support the likelihood that sensitivity to oxidative stress represents a predisposing factor common to $\mathrm{PD}$-vulnerable neuronal populations. The finding that both nigral and $\mathrm{DMnX}$ neurons display a high vulnerability to oxidative challenges is also noteworthy. A nigro-vagal pathway that controls gastric tone and motility has recently been shown to connect these 2 brain regions, raising the possibility that the pathological consequences of an oxidative injury may be relayed and possibly amplified through this anatomical and functional connection (59).

Nigral dopaminergic neurons and $\mathrm{DMnX}$ cholinergic cells share metabolic properties that could ultimately contribute to their high vulnerability to oxidative stress. In particular, a prooxidant environment characterizing both nigral and $\mathrm{DMnX}$ neurons has been proposed to arise from their reliance on calcium currents for pacemaking firing activity. The high metabolic demands associated with cytosolic calcium oscillations could stimulate mitochondrial oxidative phosphorylation, increase mitochondrial ROS production, and predispose these neurons to ROS accumulation after oxidative challenges $(4,11,50,60)$. Our present findings are therefore compatible with the interpretation that, acting on the background of this prooxidant metabolism, challenges with $\alpha$-synuclein and paraquat, alone or in combination, caused an oxidative burden within susceptible DMnX neurons.

Both intra- and extraneuronal mechanisms could contribute to an enhanced ROS production after h $\alpha$-synuclein overexpression and/or paraquat exposure. Within neurons, protein overexpression may induce ROS accumulation as a consequence of mitochondria- $\alpha$-synuclein interactions. This possibility is supported by a recent investigation revealing that modified forms of $\alpha$-synuclein, such as $\alpha$-synuclein oligomers, inhibited mitochondrial protein import by binding with high affinity to the translocase of the outer membrane 20 (TOM20) receptor; when levels of $\alpha$-synuclein were elevated, deleterious consequences of this inhibition included an impairment of mitochondrial respiration and increased ROS production (61). An important role of extraneuronal mechanisms in DMnX oxidative stress and ensuing pathology is indicated by our present results in transgenic mice lacking microglial NADPH oxidase activity. In these animals, inhibition of the NADPH oxidasedependent redox cycling of paraquat and reduced generation of microglia-derived ROS dramatically protected DMnX neurons from severe oxidative injury after combined ho-synuclein overexpression and paraquat exposure. Of note, the involvement of microglial NADPH oxidase in neuronal oxidative injury represents another feature shared by the $\mathrm{DMnX}$ and substantia nigra. Earlier investigations have indeed shown that, in models of nigrostriatal degeneration, lack of functional NADPH oxidase is associated with neuroprotection $(45,62)$.

During oxidative stress, both superoxide anion and nitric oxide are likely to be generated. In particular, within neurons with calcium-dependent pacemaking, mitochondrial calcium influx has been shown to stimulate nitric oxide synthase activity, thus promoting nitric oxide production (63). Reaction of nitric oxide with superoxide generates the peroxynitrite anion, which can readily dissociate into hydroxyl and nitrogen dioxide radicals; nitrogen dioxide directly reacts with tyrosine residues of proteins, resulting in their nitration to 3-NT (64). $\alpha$-Synuclein, with its 4 tyrosine residues, can be a target for nitration, and here, a direct relationship between oxidative stress and $\alpha$-synuclein nitration was documented in the $\mathrm{DMnX}$ using immunohistochemistry, ELISA, and a new PLA. h $\alpha$-Synuclein/3-NT PLA proved to be a highly sensitive assay to detect nitrated h $\alpha$-synuclein under conditions of milder (single treatment with h $\alpha$-synuclein AAVs) or severe (combined h $\alpha$-synuclein AAVs plus paraquat treatment) ROS production. It also allowed a quantitative assessment of the extent of $\alpha$-synuclein nitration on histological tissue sections, indicating a 2- to 3-fold increase after combined as compared with single treatment. Based on both immunohistochemical and PLA observations, mild oxidative stress was associated with a buildup of nitrated $\alpha$-synuclein mostly within dystrophic DMnX neurites; on the other hand, accumulation of nitrated $\alpha$-synuclein became overt within neuronal cell bodies only after more pronounced ROS generation. Data suggest, therefore, that neuronal projections may represent preferential and/or early sites for the pathological accumulation of nitrated $\alpha$-synuclein during oxidative challenges.

Syn 505, an antibody raised against peroxynitrite-treated $\alpha$-synuclein, recognizes both nitrated and nonnitrated $\alpha$-synuclein $(28,29)$. In line with this biochemical characterization, staining of DMnX tissue with Syn 505 produced a more robust signal than 
that generated by staining with anti-nitrated $\alpha$-synuclein nSyn 24 or with h $\alpha$-synuclein/3-NT PLA. It is noteworthy, however, that, despite its relative lack of specificity toward nitrated $\alpha$-synuclein, immunoreactivity for Syn 505 was still a sensitive indicator of oxidative stress, since labeling was more robust after a more severe ROS challenge. In the brain of patients affected by synucleinopathies, staining with Syn 505 has been shown to preferentially recognize pathological as compared with normal $\alpha$-synuclein. It detected extensive dot-like lesions in brain regions known to be targeted by Lewy inclusions as well as in regions with previously underestimated $\alpha$-synuclein pathology $(28,29)$. Based on our current findings, this distinct Syn 505 labeling of smaller pathological aggregates further supports and may actually signify an important contribution of oxidative stress to the development of $\alpha$-synuclein pathology in humans.

$\alpha$-Synuclein aggregation was also evaluated as part of this study. Protein assembly occurred within DMnX neurons of hasynuclein-overexpressing mice, but was overtly more pronounced when overexpression was followed by paraquat administration, suggesting a relationship between enhanced ROS formation and increased $\alpha$-synuclein aggregation in vivo. It is conceivable that oxidative $\alpha$-synuclein modifications, which were detected in ROS-challenged DMnX tissue, could play a significant role in this relationship. In particular, structural and conformational variants of oxidized $\alpha$-synuclein that are recognized by Syn 505 may underlie a greater propensity to protein assembly (29). Similarly, the ability of nitrated $\alpha$-synuclein to modulate pathways of protein aggregation has long been recognized and documented by in vitro studies. Results of these earlier investigations suggest that either oligomerization or fibrillation of $\alpha$-synuclein can be promoted by its nitration $(38-40,65)$. Here, we found that severe oxidative stress, as induced by combined $\alpha$-synuclein overexpression and paraquat administration, triggered early intraneuronal accumulation of predominantly oligomeric $\alpha$-synuclein species. Nitration-dependent changes in protein conformation may be stabilized by assembly into oligomers, thus contributing to this effect $(38,65)$. Furthermore, intraneuronal generation of mixed nitrated $\alpha$-synuclein species (nitrated monomers, dimers, and oligomers) as well as $\alpha$-synuclein nitration at all of its 4 tyrosine residues may favor the accumulation of oligomers as seen under our present experimental conditions $(39,40)$.

Another consideration arising from our analyses in the DMnX of AAV- and paraquat-injected animals concerns the contribution of $\alpha$-synuclein aggregation to neuronal degeneration. If it is assumed that accumulation of aggregate pathology plays a role in neuronal demise, then toxic oligomeric species would likely mediate this effect and contribute to the marked loss of DMnX neurons seen after severe oxidative stress. This conclusion is in line with increasing in vitro and in vivo experimental evidence as well as neuropathological observations supporting a deleterious potential of $\alpha$-synuclein oligomers $(42,66-68)$. Small protein aggregates may be accumulated and play a more relevant role at early stages of pathogenetic processes, including early damage after oxidative challenges $(20,42)$. Formation and accumulation of fibrillar $\alpha$-synuclein species may, on the other hand, underlie the development of more advanced pathological processes, such as prion-like $\alpha$-synuclein propagation and deposition of intraneuronal Lewy inclusions $(20,69,70)$.
In $\mathrm{PD}$, neuron-to-neuron transfer and consequent spreading of pathogenic $\alpha$-synuclein from the lower brain stem toward higher brain regions may contribute to pathological disease progression $(12,16)$. Using our models of h $\alpha$-synuclein overexpression and oxidative stress in the DMnX, we found that enhanced ROS production caused by paraquat was associated with an increased advancement of h $\alpha$-synuclein from the DMnX to the pons and then to the mouse midbrain. To the best of our knowledge, this is the first report of a distinct role of oxidative stress in ha-synuclein brain spreading. Evidence strengthening this conclusion includes the findings of rescue experiments in which enhanced spreading by paraquat was virtually abolished in mice with reduced NADPH oxidase-dependent ROS generation. These results in gp91phox-deficient mice also underscore a mechanism by which microglial cells could contribute to pathogenetic processes involving h $\alpha$-synuclein; microglial activation is likely to result in enhanced ROS production and, through this mechanism, could not only play a role in inducing oxidative stress, but also promote neuron-to-neuron transfer of pathogenic h $\alpha$-synuclein.

Caudo-rostral advancement of h $\alpha$-synuclein triggered by its overexpression in the $\mathrm{DMnX}$ is dependent upon protein transfer from medullary donor neurons into recipient axons that project into the dorsal medulla oblongata from higher brain regions (20, $21,25,43)$. A likely explanation for the present findings is therefore that oxidative stress affects neuron-to-neuron protein transmission, with increased ROS production promoting interneuronal h $\alpha$-synuclein mobility. This interpretation of the in vivo data is strongly supported by results of our in vitro experiments carried out in cocultures of cells expressing h $\alpha$-synuclein fused with V1S or SV2 Venus fragments (48). Indeed, enhanced BiFC after incubations in the presence of paraquat, hydrogen peroxide, or hydrogen peroxide plus iron sulfate indicated a higher propensity of ha-synuclein to pass from cell to cell during an oxidative challenge. It is noteworthy that increased in vitro cell crossing as well as enhanced in vivo spreading were unlikely to be a mere consequence of ho-synuclein release from damaged/dead cells. In the in vitro setting, paraquat-induced increase in BiFC occurred in the absence of any overt evidence of cytotoxicity. In mice, severe oxidative stress after cotreatment with h $\alpha$-synuclein AAVs and paraquat did cause a more pronounced neuronal death. This enhanced neurodegeneration, however, would not be expected to cause an increase in protein spreading, since earlier investigations found that overexpression-induced h $\alpha$-synuclein transfer is more efficient between relatively healthy cells and is in fact lessened after degeneration of $\mathrm{DMnX}$ neurons $(21,44)$.

Our in vivo and in vitro data support the likelihood that, following an oxidative challenge, oxidatively modified forms of h $\alpha$ synuclein that are readily transferable from cell to cell are generated. An initial clue of mobility of oxidized/nitrated ho-synuclein was provided by the in vivo observation that, following a more severe oxidative stress, increased levels of Syn 505-immunoreactive and nitrated protein were present within donor DMnX neurons as well as within recipient pontine axons. In vitro evidence then revealed that fluorescent h $\alpha$-synuclein oligomers formed as a result of bimolecular complementation within recipient cells contained nitrated h $\alpha$-synuclein. Incubations in the presence of paraquat not only promoted BiFC, but also enhanced the extent of nitration of 
the fluorescent oligomers. Finally, addition of antibodies capable of blocking nitrated $\alpha$-synuclein in the incubation medium prevented this protein exchange and markedly suppressed BiFC. Taken together, these findings suggest that, following increased production of oxidized/nitrated $\alpha$-synuclein during oxidative stress, greater amounts of modified protein were exchanged between donor and recipient cells. Oxidative modifications likely denote pathogenetic forms of $\alpha$-synuclein with pronounced conformational and structural abnormalities $(29,39,40)$. Therefore, high mobility of oxidized/nitrated $\alpha$-synuclein could result in the exchange of deleterious protein species and contribute to their propagation throughout the brain. Brain regions with higher vulnerability to oxidative stress may be at higher risk for the spreading of these pathogenetic ho-synuclein forms. Further work is warranted to determine whether a distinct pattern of connectome-dependent spreading may arise, at least in part, from tissue/cellular vulnerability to oxidative pathology. Future investigations will also establish feasibility and efficacy of therapeutic strategies that, targeting oxidized/nitrated $\alpha$-synuclein, may help counteract the progression of disease pathology in PD and other human synucleinopathies.

\section{Methods}

Viral vectors. Transgenic expression of h $\alpha$-synuclein was induced using recombinant AAVs (AAV2/6; Sirion Biotech). Gene expression was regulated by the human synapsin 1 promoter and enhanced using a woodchuck hepatitis virus posttranscriptional regulatory element and a polyadenylation signal sequence.

Animals and treatment. The majority of the experiments were conducted on male C57BL/6NRj mice (Janvier) between 12 and 16 weeks of age. For experiments aimed at determining the effects of NADPH oxidase deficiency on paraquat-induced pathology, male gp91phox-deficient mice on a C57BL/6J background strain and C57BL/6J WT animals (B6.129S-Cybb $b^{\text {tmlDin }} / \mathrm{J}$, Jackson Laboratory) were used. Animals were housed in a specific pathogen-free facility under a 12-hour light/12-hour dark cycle with ad libitum access to food and water. Paraquat dichloride hydrate (Sigma-Aldrich) was dissolved in $0.9 \%$ saline and administered i.p. at 2 doses of $15 \mathrm{mg} / \mathrm{kg}$ separated by a 1 -week interval. Animals injected with $0.9 \%$ saline served as controls. h $\alpha$-Synuclein AAVs $\left(7 \times 10^{11}\right.$ genome copies $\left./ \mathrm{ml}\right)$ were injected into the left vagus nerve as previously described (20). DHE was dissolved in DMSO (40\% in saline) and injected s.c. at a dose of $5 \mathrm{mg} / \mathrm{kg}$ 1 hour prior to sacrifice. Mice were sacrificed with an i.p. injection of $140 \mathrm{mg} / \mathrm{kg}$ sodium pentobarbital. They were then perfused through the ascending aorta with $4 \%(\mathrm{w} / \mathrm{v})$ paraformaldehyde (PFA). Brains were removed and immersed in $4 \%$ PFA for 24 hours before being cryopreserved in $30 \%(\mathrm{w} / \mathrm{v})$ sucrose. For analyses requiring nonfixed tissue (e.g., ELISA), mice were perfused with $0.9 \%$ saline, and brains were snap-frozen and stored at $-80^{\circ} \mathrm{C}$ until use.

Immunohistochemistry and image acquisition. A summary of primary antibodies and their source and working dilutions is shown in Supplemental Table 1. Coronal brain sections $(35 \mu \mathrm{m})$ were cut using a freezing microtome. Single and double labeling were carried out for fluorescence and brightfield microscopy using established protocols with a few modifications $(20,25)$. Free-floating medulla oblongata sections for double-fluorescence staining were treated with an antigen-retrieval solution $(10 \mathrm{mM}$ sodium citrate in $0.05 \%$ Tween 20 , $\mathrm{pH}=6.0$ ) at $98^{\circ} \mathrm{C}$ for 5 minutes. For nSyn24.8/h $\alpha$-synuclein immu- nolabeling, sections were incubated for an additional hour in $0.01 \%$ SDS in Tris-HCl. Endogenous mouse IgG reactivity was blocked using Mouse on Mouse (MOM) Blocking Reagent (Vector Laboratories). Following blocking in 5\% normal serum, sections were incubated with primary antibodies. ho-Synuclein antibodies were directly labeled with DyLight-594-conjugated secondary antibody (1:300). Other mouse monoclonal antibodies were labeled by a 2-step protocol using first a horse anti-mouse biotinylated secondary antibody (1:200) and then DyLight-488 streptavidin (1:300). All secondary antibodies were purchased from Vector Laboratories. In some instances, sections were counterstained with 300 nM DAPI (Thermo Fisher Scientific), a nuclear marker, prior to mounting. Sections stained with nSyn 24.8 and processed for brightfield microscopy were also treated with antigen-retrieval solution (see above) and developed with Vector SG Peroxidase Substrate Kit (Vector Laboratories). Fluorescence images were collected on Zeiss microscopes (LSM700 or LSM800) with a $\times 63$ Plan-Apochromat objective. They were processed and analyzed with Fiji software (ImageJ, NIH, version 2.0.0) unless otherwise specified. A mouse brain atlas was used as reference for brain coordinates (71).

$O x$-DHE and MDA quantification. Ox-DHE fluorescent signal was acquired from ChAT-immunoreactive neurons in the DMnX (bregma -7.48 to $-7.32 \mathrm{~mm})$, hypoglossal nucleus $(-7.48$ to $-7.32 \mathrm{~mm})$, striatum $(+0.26$ to $+1.10 \mathrm{~mm})$, and medial septal nucleus $(+0.26$ to $+1.10 \mathrm{~mm})$ or from h $\alpha$-synuclein-immunoreactive DMnX neurons. Using confocal images, neurons were delineated based on ChAT or h $\alpha$-synuclein immunoreactivity. Ox-DHE puncta within the delineated cells were selected by applying a constant intensity threshold and outlined using the "analyze particles" function. The total integrated density of the outlined puncta was quantified for each image, divided by the number of neurons, and averaged for each animal. For quantification of MDA, brains were removed and snap-frozen on dry ice. Specimens of the dorso-medial medulla oblongata (10 mg wet tissue/animal) were assayed using a Colorimetric MDA Assay Kit (Abcam) according to the manufacturer's protocol.

Quantification of transduction. Investigators performing histological analyses were blinded to sample treatment. AAV-induced transduction was estimated from confocal images of medulla oblongata sections immunostained for h $\alpha$-synuclein (MJFR1). Every fifth section of the entire left DMnX between bregma -7.32 and $-7.20 \mathrm{~mm}$ was acquired with a $\times 20$ objective. The total number of h $\alpha$-synuclein-positive and DAPI-stained neurons was counted using the cell counter plugin.

Counting of DMnX neurons. The number of Nissl-stained neurons was quantified throughout the entire $\mathrm{DMnX}$ using every fifth section. Stereological counts were performed on an IX2 UCB Olympus microscope using an optical fractionator (Stereo Investigator, version 9, MBF Bioscience). Coefficients of error were less than 0.10.

Quantification of Syn 505 fluorescence. Fluorescence intensity was measured in DMnX-containing tissue sections immunostained with Syn 505 antibody and anti-h $\alpha$-synuclein (15G7). Every fifth medulla oblongata section between bregma -7.48 and $-7.20 \mathrm{~mm}$ was used to acquire single-plane images of the left DMnX. For each image, a Gaussian blur and background subtraction were applied. An intensity threshold was also selected to detect and outline 15G7-positive structures. The median fluorescence of either 15G7 or Syn 505 labeling was measured. Data were calculated as the median ratio of Syn 505 over $15 G 7$ fluorescence. Fluorescence intensity was also quantified within pontine axons immunolabeled with Syn 505 and 15G7. Confocal 
images taken within the locus coeruleus or parabrachial nucleus between bregma -5.68 and $-5.34 \mathrm{~mm}$ were used for these analyses. Each axon was outlined based on a constant fluorescence threshold for 15G7 immunoreactivity. The mean fluorescence intensity of Syn 505 labeling was measured within the delineated area and corrected for background fluorescence.

Axonal counts and quantification of spreading. The total number of h $\alpha$-synuclein-immunoreactive (Syn211) axons was counted in sections of the left pons (bregma $-5.40 \mathrm{~mm}$ ) and the left caudal (bregma -4.60 $\mathrm{mm}$ ) and rostral (bregma $-3.40 \mathrm{~mm}$ ) midbrain. Measurements of axonal length and density were carried out in pontine sections (bregma $-5.68,-5.51$, and $-5.34 \mathrm{~mm}$ ) after delineation of an area encompassing the locus coeruleus and parabrachial nucleus, using the Spaceballs stereological probe (Stereo Investigator software, version 9, MBF Biosciences) (20). High-magnification images of Syn211-immunoreactive axons were acquired on an Observer.Z1 microscope (Zeiss), using a $\times 63$ Plan-Apochromat objective.

PLA. PLA was performed using Duolink (Sigma-Aldrich) according to the manufacturer's protocols. Aggregated ho-synuclein was detected using PLA probes conjugated to a h $\alpha$-synuclein-specific antibody (Syn211), as previously described (20, 42). Nitrated hosynuclein was detected by either direct or indirect PLA. For direct PLA, PLUS and MINUS oligonucleotide probes were conjugated with a hosynuclein antibody (MJFR1) and anti-3-NT, respectively (Duolink Probemaker, Sigma-Aldrich). For indirect PLA, sections were first incubated overnight in primary antibodies (3-NT and MJFR1) and then incubated with secondary antibodies conjugated with oligonucleotide probes, i.e., anti-rabbit PLUS and anti-mouse MINUS (SigmaAldrich). Following ligation and amplification, specific PLA signals were visualized using brightfield or fluorescence detection kits (Duolink, Sigma-Aldrich). In some instances, sections were counterstained with hematoxylin or DAPI. For PLA/immunohistochemistry double labeling, the PLA signal was detected using the Duolink Green Detection Kit (Sigma-Aldrich). Tissue sections were then immunolabeled for ho-synuclein (4B12) using DyLight-594 as a secondary antibody. In SH-SY5Y cells, the PLA signal was visualized using the Duolink Red Fluorescence Detection Kit (Sigma-Aldrich). Specificity of ho-synuclein/3-NT PLA was confirmed by experiments in which medulla oblongata sections were first incubated with a cocktail of concentrated monoclonal antibodies raised against nitrated $\alpha$-synuclein (nSyn12, nSyn14, and nSyn24.8) for 48 hours at $4^{\circ} \mathrm{C}$. These sections were then processed for direct h $\alpha$-synuclein/3-NT PLA, as described above. Preincubation with nitrated $\alpha$-synuclein antibodies completely abolished the PLA signal (Supplemental Figure 1). For quantification of the syn/syn and ho-synuclein/3-NT PLA signals in the DMnX, medullary sections encompassing the entire DMnX were used. The DMnX was delineated at low magnification ( $\times 10$ objective) on every tenth section (between bregma -6.96 and $-8.00 \mathrm{~mm}$ ), and the number of PLA dots was counted stereologically. Coefficients of error were less than 0.10. Analysis of h $\alpha$-synuclein/3-NT PLA dots in the pons was performed on a single section corresponding to bregma $-5.34 \mathrm{~mm}$. Counts were performed in the entire left hemisphere using the meander scan function of Stereo Investigator (MBF Bioscience).

ELISA. For measurements of nitrated $\alpha$-synuclein in vivo, the left dorso-medial medulla oblongata was dissected under a stereomicroscope and sonicated in RIPA buffer $(\times 10 \mathrm{w} / \mathrm{v})$ with protease inhibitors (Sigma-Aldrich). Tissue homogenates were centrifuged at $16,000 \mathrm{~g}$ for 15 minutes at $4^{\circ} \mathrm{C}$. Protein concentration was determined from the supernatants by BCA assay (Pierce Biotechnology). 96-Well ELISA plates (MaxiSorp, Nunc) coated with $1 \mu \mathrm{g} / \mathrm{ml}$ of the capture antibody, i.e., anti- $\alpha$-synuclein (Syn-1) in $50 \mathrm{mM}$ carbonate buffer ( $\mathrm{pH}$ 9.6), were washed with PBS containing 0.05\% Tween 20 and blocked with SuperBlock T20 (Thermo Fisher Scientific) for 1 hour at room temperature. Samples ( $0.1 \mathrm{mg}$ protein) were added to the wells and incubated at room temperature for 2.5 hours with shaking. After washing, further incubations were carried out first with the biotinylated detection antibody, i.e., 3-NT $(1 \mu \mathrm{g} / \mathrm{ml})$, for 1.5 hours and then with avidin-conjugated peroxidase (ExtrAvidin; SigmaAldrich). Final steps of the protocol involved subsequent additions of 3,3'5,5'-tetramethylbenzidine solution (Sigma-Aldrich) and $2 \mathrm{~N}$ sulfuric acid. Absorbance was measured at $450 \mathrm{~nm}$ using an Anthos 2010 plate reader (Biochrome Ltd.). For measurements of h $\alpha$ synuclein in vitro, cells were lysed in RIPA buffer. Syn-1 was used as capture antibody. Coating of 96-well plates and washing procedures were similar to those described above. Samples $(20 \mu \mathrm{g}$ protein with $1 \%$ SDS) and recombinant h $\alpha$-synuclein monomers (for generation of a standard curve) were added to the wells. MJFR1 antibody (1 $\mu \mathrm{g} /$ $\mathrm{ml}$ ) was then used for detection together with horseradish peroxidase-conjugated anti-rabbit IgG (1:1000; Promega).

In vitro experiments/analyses. SH-SY5Y human neuroblastoma cell lines stably expressing the N-terminal half of Venus YFP-tagged h $\alpha$-synuclein (V1S) or the C-terminal half of Venus YFP-tagged h $\alpha$ synuclein (SV2) were cocultured in equivalent numbers and maintained as previously described (48). Paraquat, hydrogen peroxide, and iron sulfate were dissolved in sterile PBS or distilled water. To determine cell viability, cells were trypsinized and stained with acridine orange/propidium iodide (Logos Biosystems) prior to automated cell counting (LUNA-FLTM Dual Fluorescence Cell Counter). All other analyses were made on cells grown on poly-L-lysine-coated coverslips and fixed in 4\% PFA. Nuclei were stained with DAPI. Assessment of BiFC fluorescence and quantification of in vitro ho-synuclein/ 3-NT PLA were carried out on confocal stack images acquired on a Zeiss LSM710 microscope (BiFC) or a Zeiss LSM800 microscope with airy scan (PLA) under a $\times 63$ Apochrome objective. The number of both total and BiFC-positive cells was counted using the cell counter plugin (48). For assessment of BiFC-integrated density, the intracellular fluorescent signal was measured after delineation the BiFC-positive cells. The number of PLA dots per cell was counted on representative images randomly acquired from multiple areas on the coverslip. Counts were made using the spots function of the Imaris software (version 8) and averaged over the number of DAPI-stained nuclei. The number of BiFC aggregates with or without PLA dots was counted on the entire coverslip to calculate the percentage of colocalization. In experiments in which antibodies were added to the incubation medium, IgG, anti-3NT, or nSyn12 was used at a concentration of $50 \mu \mathrm{g} / \mathrm{ml}$.

Statistics. Analyses were performed with GraphPad Prism (8.0) or R software (3.5.2) using nonparametric Mann-Whitney $U$ test for comparisons between 2 groups. Comparisons among more than 2 groups were carried out using Kruskal-Wallis 1-way ANOVA followed by Dunn's or Conover-Iman post hoc tests. $P$ values of less than 0.05 were considered statistically significant.

Study approval. Animal experiments were approved by the State Agency for Nature, Environment, and Consumer Protection in North Rhine Westphalia, Germany. 


\section{Author contributions}

REM designed and performed in vivo experiments and analyzed data. $\mathrm{MH}$ performed surgical procedures and contributed to histological quantifications. EJB designed and performed in vitro experiments and analyzed their results. HA contributed to analysis and quantification of the in vitro work. AU carried out PLA and other histological evaluations. SJL, AU, and DADM supervised different aspects of the project. DADM designed experiments, analyzed data, and wrote the manuscript with input from the other authors.

\section{Acknowledgments}

The authors thank Sarah A. Jewell for her comments on the manuscript, Omar El-Agnaf (Qatar Biomedical Research Institute) for kindly providing conformation-specific antibodies, Celine
Galvagnion for the production of $\alpha$-synuclein standards, Bettina Winzen-Reichert and Laura Jakobi for assistance with the experiments, and personnel at the DZNE Light Microscope Facility, Image and Data Analysis Facility, and Preclinical Center. This work was supported by the Paul Foundation, the EU Joint Programme-Neurodegenerative Disease (JPND 01ED1612) and National Research Foundation (NRF) grants by the Korean Government (Ministry of Science, ICT, and Future Planning [MSIP]; NRF2018R1A2A1A05078261 and NRF-2018R1A5A2025964).

Address correspondence to: Donato A. Di Monte, German Center for Neurodegenerative Diseases, Venusberg-Campus 1, Building 99, 53127 Bonn, Germany. Phone: 49.228.43302650; Email: donato.dimonte@dzne.de.
1. Graham DG. Oxidative pathways for catecholamines in the genesis of neuromelanin and cytotoxic quinones. MolPharmacol.1978;14(4):633-643.

2. Sulzer D. Multiple hit hypotheses for dopamine neuron loss in Parkinson's disease. Trends Neurosci. 2007;30(5):244-250.

3. Chan CS, et al. 'Rejuvenation' protects neurons in mouse models of Parkinson's disease. Nature. 2007;447(7148):1081-1086.

4. Guzman JN, et al. Oxidant stress evoked by pacemaking in dopaminergic neurons is attenuated by DJ-1. Nature. 2010;468(7324):696-700.

5. Burbulla LF, et al. Dopamine oxidation mediates mitochondrial and lysosomal dysfunction in Parkinson's disease. Science. 2017;357(6357):1255-1261.

6. Dryanovski DI, et al. Calcium entry and $\alpha$-synuclein inclusions elevate dendritic mitochondrial oxidant stress in dopaminergic neurons. J Neurosci. 2013;33(24):10154-10164.

7. Tapias V, Hu X, Luk KC, Sanders LH, Lee VM, Greenamyre JT. Synthetic alpha-synuclein fibrils cause mitochondrial impairment and selective dopamine neurodegeneration in part via iNOS-mediated nitric oxide production. Cell Mol Life Sci. 2017;74(15):2851-2874.

8. Eadie MJ. The pathology of certain medullary nuclei in parkinsonism. Brain. 1963;86:781-792.

9. Halliday GM, Blumbergs PC, Cotton RG, Blessing WW, Geffen LB. Loss of brainstem serotoninand substance P-containing neurons in Parkinson's disease. Brain Res. 1990;510(1):104-107.

10. Gai WP, Blumbergs PC, Geffen LB, Blessing WW. Age-related loss of dorsal vagal neurons in Parkinson's disease. Neurology. 1992;42(11):2106-2111.

11. Goldberg JA, et al. Calcium entry induces mitochondrial oxidant stress in vagal neurons at risk in Parkinson's disease. Nat Neurosci. 2012;15(10):1414-1421.

12. Braak H, Del Tredici K, Rüb U, de Vos RA, Jansen Steur EN, Braak E. Staging of brain pathology related to sporadic Parkinson's disease. Neurobiol Aging. 2003;24(2):197-211.

13. Kordower JH, Chu Y, Hauser RA, Freeman TB, Olanow CW. Lewy body-like pathology in longterm embryonic nigral transplants in Parkinson's disease. Nat Med. 2008;14(5):504-506.

14. Desplats P, et al. Inclusion formation and neuronal cell death through neuron-to-neuron trans- mission of alpha-synuclein. Proc Natl Acad Sci US A. 2009;106(31):13010-13015.

15. Freundt EC, et al. Neuron-to-neuron transmission of $\alpha$-synuclein fibrils through axonal transport. Ann Neurol. 2012;72(4):517-524.

16. Goedert M, Spillantini MG, Del Tredici K, Braak H. 100 years of Lewy pathology. Nat Rev Neurol. 2013;9(1):13-24.

17. Holmqvist S, et al. Direct evidence of Parkinson pathology spread from the gastrointestinal tract to the brain in rats. Acta Neuropathol. 2014;128(6):805-820.

18. Svensson E, et al. Vagotomy and subsequent risk of Parkinson's disease. Ann Neurol. 2015;78(4):522-529.

19. Ulusoy A, Phillips RJ, Helwig M, Klinkenberg M, Powley TL, Di Monte DA. Brain-to-stomach transfer of $\alpha$-synuclein via vagal preganglionic projections. Acta Neuropathol. 2017;133(3):381-393.

20. Helwig M, et al. Brain propagation of transduced $\alpha$-synuclein involves non-fibrillar protein species and is enhanced in $\alpha$-synuclein null mice. Brain. 2016;139(Pt 3):856-870.

21. Rusconi R, Ulusoy A, Aboutalebi H, Di Monte DA. Long-lasting pathological consequences of overexpression-induced $\alpha$-synuclein spreading in the rat brain. Aging Cell. 2018;17(2):e12727.

22. Di Monte DA. The environment and Parkinson's disease: is the nigrostriatal system preferentially targeted by neurotoxins? Lancet Neurol. 2003;2(9):531-538.

23. Budd SL, Castilho RF, Nicholls DG. Mitochondrial membrane potential and hydroethidinemonitored superoxide generation in cultured cerebellar granule cells. FEBS Lett. 1997;415(1):21-24.

24. Andrews ZB, et al. Uncoupling protein-2 is critical for nigral dopamine cell survival in a mouse model of Parkinson's disease. J Neurosci. 2005;25(1):184-191.

25. Ulusoy A, et al. Caudo-rostral brain spreading of $\alpha$-synuclein through vagal connections. EMBO Mol Med. 2013;5(7):1119-1127.

26. Giasson BI, et al. Oxidative damage linked to neurodegeneration by selective alpha-synuclein nitration in synucleinopathy lesions. Science. 2000;290(5493):985-989.

27. Duda JE, et al. Widespread nitration of pathological inclusions in neurodegenerative synucleinopathies. Am J Pathol. 2000;157(5):1439-1445.
28. Duda JE, Giasson BI, Mabon ME, Lee VM, Trojanowski JQ. Novel antibodies to synuclein show abundant striatal pathology in Lewy body diseases. Ann Neurol. 2002;52(2):205-210.

29. Waxman EA, Duda JE, Giasson BI. Characterization of antibodies that selectively detect alpha-synuclein in pathological inclusions. Acta Neuropathol. 2008;116(1):37-46.

30. Elfineh L, Classon C, Asplund A, Pettersson U, Kamali-Moghaddam M, Lind SB. Tyrosine phosphorylation profiling via in situ proximity ligation assay. BMC Cancer. 2014;14:435.

31. Sahin U, Jollivet F, Berthier C, de Thé H, Lallemand-Breitenbach V. Detection of protein SUMOylation in situ by proximity ligation assays. Methods Mol Biol. 2016;1475:139-150.

32. Avin A, Levy M, Porat Z, Abramson J. Quantitative analysis of protein-protein interactions and post-translational modifications in rare immune populations. Nat Commun. 2017;8(1):1524.

33. Hashimoto $\mathrm{M}$, et al. Oxidative stress induces amyloid-like aggregate formation of NACP/ alpha-synuclein in vitro. Neuroreport. 1999;10(4):717-721.

34. Ischiropoulos $\mathrm{H}$, Beckman JS. Oxidative stress and nitration in neurodegeneration: cause, effect, or association? J Clin Invest. 2003;111(2):163-169.

35. Brahmachari S, et al. Activation of tyrosine kinase c-Abl contributes to $\alpha$-synuclein-induced neurodegeneration. JClin Invest. 2016;126(8):2970-2988.

36. Scudamore O, Ciossek T. Increased oxidative stress exacerbates $\alpha$-synuclein aggregation in vivo. $\mathrm{JNeu}$ ropathol Exp Neurol. 2018;77(6):443-453.

37. Paxinou E, et al. Induction of alpha-synuclein aggregation by intracellular nitrative insult. JNeurosci. 2001;21(20):8053-8061.

38. Uversky VN, et al. Effects of nitration on the structure and aggregation of alpha-synuclein. Brain Res Mol Brain Res. 2005;134(1):84-102.

39. Hodara R, et al. Functional consequences of alpha-synuclein tyrosine nitration: diminished binding to lipid vesicles and increased fibril formation. J Biol Chem. 2004;279(46):47746-47753.

40. Burai R, Ait-Bouziad N, Chiki A, Lashuel HA. Elucidating the role of site-specific nitration of $\alpha$-synuclein in the pathogenesis of Parkinson's disease via protein semisynthesis and mutagenesis. J Am Chem Soc. 2015;137(15):5041-5052. 
41. Vaikath NN, et al. Generation and characterization of novel conformation-specific monoclona antibodies for $\alpha$-synuclein pathology. Neurobiol Dis. 2015;79:81-99.

42. Roberts RF, Wade-Martins R, Alegre-Abarrategui J. Direct visualization of alpha-synuclein oligomers reveals previously undetected pathology in Parkinson's disease brain. Brain. 2015;138 (Pt 6):1642-1657.

43. Bae EJ, et al. LRRK2 kinase regulates $\alpha$-synuclein propagation via RAB35 phosphorylation. Nat Commun. 2018;9(1):3465.

44. Ulusoy A, et al. Neuron-to-neuron $\alpha$-synuclein propagation in vivo is independent of neuronal injury. Acta Neuropathol Commun. 2015;3:13.

45. Purisai MG, McCormack AL, Cumine S, Li J, Isla MZ, Di Monte DA. Microglial activation as a priming event leading to paraquat-induced dopaminergic cell degeneration. Neurobiol Dis. 2007;25(2):392-400.

46. Bonneh-Barkay D, Reaney SH, Langston WJ, Di Monte DA. Redox cycling of the herbicide paraquat in microglial cultures. Brain Res $\mathrm{Mol}$ Brain Res. 2005;134(1):52-56.

47. Pollock JD, et al. Mouse model of X-linked chronic granulomatous disease, an inherited defect in phagocyte superoxide production. Nat Genet. 1995;9(2):202-209.

48. Bae EJ, et al. Glucocerebrosidase depletion enhances cell-to-cell transmission of $\alpha$-synuclein. Nat Commun. 2014;5:4755.

49. Schapira AH. Mitochondria in the aetiology and pathogenesis of Parkinson's disease. Lancet Neurol. 2008;7(1):97-109.

50. Surmeier DJ, Obeso JA, Halliday GM. Selective neuronal vulnerability in Parkinson disease. Nat Rev Neurosci. 2017;18(2):101-113.

51. Heneka MT, McManus RM, Latz E. Inflammasome signalling in brain function and neurodegenerative disease. Nat Rev Neurosci.
2018;19(10):610-621.

52. McCormack AL, Atienza JG, Langston JW, Di Monte DA. Decreased susceptibility to oxidative stress underlies the resistance of specific dopaminergic cell populations to paraquat-induced degeneration. Neuroscience. 2006;141(2):929-937.

53. Mosharov EV, et al. Interplay between cytosolic dopamine, calcium, and alpha-synuclein causes selective death of substantia nigra neurons. Neuron. 2009;62(2):218-229.

54. Hirsch E, Graybiel AM, Agid YA. Melanized dopaminergic neurons are differentially susceptible to degeneration in Parkinson's disease. Nature. 1988;334(6180):345-348.

55. German DC, Manaye KF, Sonsalla PK, Brooks BA. Midbrain dopaminergic cell loss in Parkinson's disease and MPTP-induced parkinsonism: sparing of calbindin-D28k-containing cells. Ann N Y Acad Sci. 1992;648:42-62.

56. Seidel K, et al. The brainstem pathologies of Parkinson's disease and dementia with Lewy bodies. Brain Pathol. 2015;25(2):121-135.

57. Mori F, Tanji K, Zhang H, Kakita A, Takahashi H, Wakabayashi K. alpha-Synuclein pathology in the neostriatum in Parkinson's disease. Acta Neuropathol. 2008;115(4):453-459.

58. Hall H, et al. Hippocampal Lewy pathology and cholinergic dysfunction are associated with dementia in Parkinson's disease. Brain. 2014;137(Pt 9):2493-2508.

59. Anselmi L, Toti L, Bove C, Hampton J, Travagli RA. A nigro-vagal pathway controls gastric motility and is affected in a rat model of Parkinsonism. Gastroenterology. 2017;153(6):1581-1593.

60. Guzman JN, et al. Systemic isradipine treatment diminishes calcium-dependent mitochondrial oxidant stress. J Clin Invest. 2018;128(6):2266-2280.

61. Di Maio R, et al. $\alpha$-Synuclein binds to TOM20 and inhibits mitochondrial protein import in Parkinson's disease. Sci Transl Med. 2016;8(342):342ra78

62. Wu DC, et al. NADPH oxidase mediates oxidative stress in the 1-methyl-4-phenyl-1,2,3,6-tetrahydropyridine model of Parkinson's disease. Proc Natl Acad Sci USA . 2003;100(10):6145-6150.

63. Sanchez-Padilla J, et al. Mitochondrial oxidant stress in locus coeruleus is regulated by activity and nitric oxide synthase. Nat Neurosci. 2014;17(6):832-840.

64. Schildknecht S, et al. Oxidative and nitrative alpha-synuclein modifications and proteostatic stress: implications for disease mechanisms and interventions in synucleinopathies. JNeurochem. 2013;125(4):491-511.

65. Yamin G, Uversky VN, Fink AL. Nitration inhibits fibrillation of human alpha-synuclein in vitro by formation of soluble oligomers. FEBS Lett. 2003;542(1-3):147-152.

66. Rockenstein E, et al. Accumulation of oligomerprone $\alpha$-synuclein exacerbates synaptic and neuronal degeneration in vivo. Brain. 2014;137 (Pt 5):1496-1513.

67. Deas E, et al. Alpha-synuclein oligomers interact with metal ions to induce oxidative stress and neuronal death in Parkinson's disease. Antioxid Redox Signal. 2016;24(7):376-391.

68. Mor DE, et al. Dopamine induces soluble $\alpha$-synuclein oligomers and nigrostriatal degeneration. Nat Neurosci. 2017;20(11):1560-1568.

69. Luk KC, et al. Pathological $\alpha$-synuclein transmission initiates Parkinson-like neurodegeneration in nontransgenic mice. Science. 2012;338(6109):949-953.

70. Mao X, et al. Pathological $\alpha$-synuclein transmission initiated by binding lymphocyte-activation gene 3. Science. 2016;353(6307):aah3374.

71. Franklin KBJ, Paxinos G. Paxinos and Franklin's the mouse brain in stereotaxic coordinates. 4 th ed. Amsterdam, the Netherlands: Academic Press; 2013. 